ARTICLE

\title{
APOE4 exacerbates synapse loss and neurodegeneration in Alzheimer's disease patient iPSC-derived cerebral organoids
}

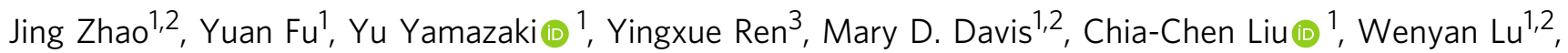
Xue Wang ${ }^{3}$, Kai Chen', Yesesri Cherukuri ${ }^{3}$, Lin Jia1, Yuka A. Martens (D) 1, Lucy Job ${ }^{1,2}$, Francis Shue (D) 1, Thanh Thanh Nguyen (D) ${ }^{1}$, Steven G. Younkin', Neill R. Graff-Radford ${ }^{4}$, Zbigniew K. Wszolek ${ }^{4}$, David A. Brafman ${ }^{5}$,

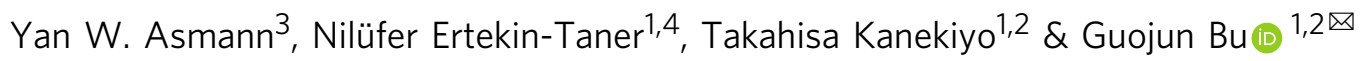

APOE4 is the strongest genetic risk factor associated with late-onset Alzheimer's disease (AD). To address the underlying mechanism, we develop cerebral organoid models using induced pluripotent stem cells (iPSCs) with $A P O E \varepsilon 3 / \varepsilon 3$ or $\varepsilon 4 / \varepsilon 4$ genotype from individuals with either normal cognition or $A D$ dementia. Cerebral organoids from $A D$ patients carrying $A P O E \& 4 / \varepsilon 4$ show greater apoptosis and decreased synaptic integrity. While AD patientderived cerebral organoids have increased levels of $A \beta$ and phosphorylated tau compared to healthy subject-derived cerebral organoids, APOE4 exacerbates tau pathology in both healthy subject-derived and AD patient-derived organoids. Transcriptomics analysis by RNAsequencing reveals that cerebral organoids from $A D$ patients are associated with an enhancement of stress granules and disrupted RNA metabolism. Importantly, isogenic conversion of $A P O E 4$ to $A P O E 3$ attenuates the APOE4-related phenotypes in cerebral organoids from AD patients. Together, our study using human iPSC-organoids recapitulates APOE4related phenotypes and suggests $A P O E 4$-related degenerative pathways contributing to $A D$ pathogenesis.

\footnotetext{
${ }^{1}$ Department of Neuroscience, Mayo Clinic, Jacksonville, FL 32224, USA. ${ }^{2}$ Center for Regenerative Medicine, Neuroregeneration Lab, Mayo Clinic, Jacksonville, FL 32224, USA. ${ }^{3}$ Department of Health Sciences Research, Mayo Clinic, Jacksonville, FL 32224, USA. ${ }^{4}$ Department of Neurology, Mayo Clinic, Jacksonville, FL 32224, USA. ${ }^{5}$ School of Biological and Health Systems Engineering, Arizona State University, Tempe, AZ 85287, USA. ${ }^{凶}$ email: bu.guojun@mayo.edu
} 
A lzheimer's disease $(\mathrm{AD})$ characterized by progressive neurodegeneration is the most common form of dementia ${ }^{1}$. While formations of senile plaques and neurofibrillary tangles (NFTs) are two major neuropathological hallmarks in $\mathrm{AD}$, these pathologies composed of amyloid- $\beta$ peptides $(A \beta)$ and phosphorylated tau often precede the onset of symptomatic dementia by decades ${ }^{2,3}$.

The $\varepsilon 4$ allele of $A P O E$ gene (APOE4) is the strongest genetic risk factor for $\mathrm{AD}$ among the three polymorphic alleles (APOE2, $A P O E 3$, and $A P O E 4)^{4,5}$. Apolipoprotein $\mathrm{E}$ (apoE) is produced primarily by astrocytes in the central nervous system as a carrier of cholesterol and other lipids to support membrane homeostasis, synaptic integrity, and injury repair ${ }^{6,7}$. Increasing evidences from animal models and postmortem human brains have shown that APOE4 is associated with multiple aspects of $\mathrm{AD}$ pathogenesis ${ }^{8}$. In particular, mouse models carrying human APOE4 have accelerated $A \beta$ seeding and suppressed $A \beta$ clearance, as well as disturbed synaptic plasticity and blood-brain barrier integrity ${ }^{7,9}$; however, whether these findings are translatable to humans is not clear. In addition, studies with human postmortem brains represent only the characteristics of end-stage disease ${ }^{10-12}$. Thus, there is an urgent need to establish human-relevant models to define APOE4-related pathogenic pathways in $\mathrm{AD}$.

The induced pluripotent stem cells (iPSCs) derived from human somatic cells with AD-linked mutations or risk alleles are promising in vitro models by recapitulating the earliest molecular and pathological changes in age-related disorders ${ }^{13-17}$. Elevated levels of $\mathrm{A} \beta$ and phosphorylated tau, as well as increased cellular stress markers have been reported in iPSC-derived neurons from $\mathrm{AD}$ patients ${ }^{14-16,18}$. For example, $\mathrm{A} \beta$ oligomers accumulate in iPSC-derived neurons and astrocytes in cells from patients with a familial amyloid precursor protein $(A P P)$ p.E693 $\triangle$ mutation and sporadic $\mathrm{AD}$, leading to endoplasmic reticulum (ER) and oxidative stress ${ }^{15}$. In addition to altered APP processing, an increase in levels of total and phosphorylated tau is observed in iPSC-derived neurons from $\mathrm{AD}$ patients carrying the $A P P \mathrm{p}$.V717I mutation or $A P O E 4$ risk allele ${ }^{18,19}$. However, the relevance of the observations from two-dimensional (2-D) cell cultures to AD is questionable as $\mathrm{AD}$ pathology is intricate and involves diverse cell types, tissue structures, and cellular pathways. Thus, in this study, we utilize a large number of human iPSC lines from healthy subjects and $\mathrm{AD}$ patients carrying $A P O E \varepsilon 3 / \varepsilon 3$ or $\varepsilon 4 / \varepsilon 4$ genotype, and investigate $\mathrm{AD}$-related phenotypes using the iPSC-derived three-dimensional (3-D) cerebral organoid model system, which is highly reminiscent of human brain structure with diverse cell types ${ }^{20-22}$. Here, we show that APOE4 aggravates neurodegeneration in iPSCderived cerebral organoids from $\mathrm{AD}$ patients; however, its effects on tauopathy are significant in both healthy subject-derived and $\mathrm{AD}$ patient-derived cerebral organoids. Interestingly, the levels of $\mathrm{A} \beta$ are increased in $\mathrm{AD}$ organoids independent of $A P O E$ genotype. Importantly, isogenic conversion of APOE4 to APOE3 attenuates $\mathrm{AD}$-related phenotypes in iPSC-derived cerebral organoids. These findings reveal APOE4-related pathways in 3-D models that are directly relevant to humans.

\section{Results}

Characterization of human iPSC-derived cerebral organoids. To study the effects of APOE genotype on AD-related pathways in a physiologically relevant environment, we generated 3-D cerebral organoid models using human iPSC lines from cognitively unimpaired individuals carrying $A P O E \varepsilon 3 / \varepsilon 3$ (Con-E3; $N=$ 5), cognitively unimpaired individuals carrying $A P O E \varepsilon 4 / \varepsilon 4$ (Con-E4; $N=5$ ), $\mathrm{AD}$ patients carrying $A P O E \varepsilon 3 / \varepsilon 3$ (AD-E3; $N=5)$ and $\mathrm{AD}$ patients carrying $A P O E \varepsilon 4 / \varepsilon 4(\mathrm{AD}-\mathrm{E} 4 ; N=5)$, collected from multiple sources (Supplementary Table 1).
Among them, 5 iPSCs lines were generated de novo from fibroblasts by transfecting with three episomal vectors encoding five transcription factors (OCT3/4, SOX2, L-MYC, KLF4, and LIN28) and p53 shRNA ${ }^{23}$. Expression of pluripotency stem cell-specific markers was confirmed by immunostainings for SSEA4, Nanog, and TRA-1-60 (Supplementary Fig. 1A). The pluripotency of the iPSC lines was also validated by their ability to differentiate into endodermal, mesodermal, and ectodermal origin cells upon immunostaining of Brachyury (Mesoderm marker), Sox17 (endoderm marker), and Nestin/Sox2 (ectoderm marker) (Supplementary Fig. 1B). The iPSC lines maintained a normal karyotype after reprogramming (Supplementary Fig. 1C). All other iPSC lines utilized in published studies ${ }^{19,24-26}$ or from California Institute for Regenerative Medicine (CIRM) (FUJIFILM Cellular Dynamics, Inc.) have been fully validated and characterized previously.

To generate cerebral organoids from iPSCs with efficiency and reproducibility, we followed an optimized protocol developed by Stemcell Technologies. Cerebral organoid formation was initiated through an intermediate embryonic body (EB) formation step followed by expansion of neuroepithelia in a matrigel scaffold. On day 12, the iPSC-derived organoids were transferred to an orbital shaker in neuronal differentiation medium and maintained under rotary conditions for maturation (Fig. 1a). On week 4, cerebral organoids showed a predominantly dorsal forebrain region specification, containing fluid-filled ventricle-like structures aligned with Sox2-positive neural progenitors in a ventricular/ subventricular-like zone (VZ/SVZ) and beta-tubulin III (Tuj1)positive neuroblasts in an outer layer (Fig. 1b). A deep cortical layer marker Ctip2-positive neurons were detected as early as week 4 (Fig. 1c), whereas a superficial cortical layer marker Satb2positive neurons emerged in the later stage at week 12 (Fig. 1d). These observations revealed the sequential emergence of different neuronal layers along with the differentiation, which is consistent with previous publication ${ }^{20}$. Since apoE is mainly produced by astrocytes in the brain ${ }^{27}$, we assessed the presence of astrocytes at different time points by immunostaining for glial fibrillary acidic protein (GFAP). We found that small clusters of GFAP-positive astrocytes started to emerge in some $\mathrm{VZ}$ area at week 4. GFAPpositive astrocytes showed an immature morphology with short processes, which were separated from surrounding Tuj1-positive neuronal cells (Fig. 1e). At week 12 of differentiation, GFAPpositive astrocytes increased in number and migrated within the neuronal layers, displaying typical mature astrocyte morphology with long processes (Fig. 1f). Furthermore, to determine the influence of the technical difference to the differentiation of cerebral organoids across different rounds, we compared the levels of GFAP and Tuj1 in the cerebral organoids from two rounds of differentiation. We found no significant differences in the expression of GFAP and Tuj1 at week 12 between the first and second round of experiments (Supplementary Fig. 9). These results indicate the successful development of iPSCs to cerebral organoids with cortical like organization composed of abundant mature neurons and astrocytes.

APOE4 and AD status exacerbate neurodegeneration. With the establishment of the cerebral organoid culture system, we generated four groups of iPSC-derived cerebral organoids (Con-E3, Con-E4, AD-E3, and AD-E4). Cerebral organoid size was monitored and there were no evident differences observed among different groups at 2 and 12 weeks (Supplementary Fig. 2). To evaluate the effect of APOE4 and disease status on neuronal apoptosis/degeneration in the cerebral organoids, cleaved caspase-3 (CASP3) was analyzed by immunostaining at week 12 (Fig. 2a). To avoid the possible influences of necrosis observed in 

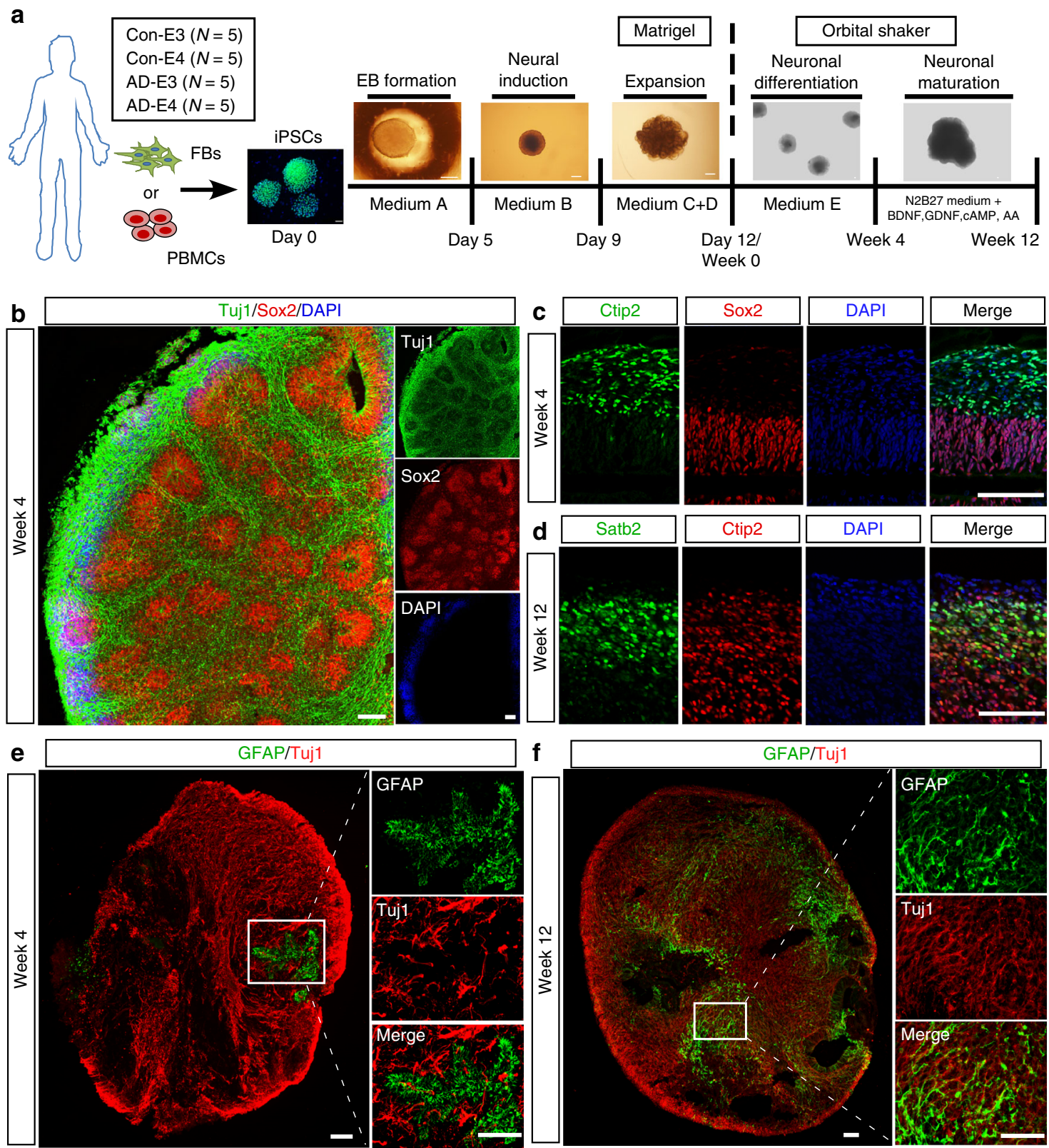

Fig. 1 Generation and characterization of cerebral organoids from human iPSCs. a A schematic overview of the procedures for generating cerebral organoids from human iPSCs using the STEMdiff ${ }^{\top M}$ Cerebral Organoid Kit. b Representative images of the ventricular zone (VZ)-like structure formed by new-born neurons (Tuj1, green) and neural progenitor cells (Sox2, red) in cerebral organoids at week 4 of differentiation. c, $\mathbf{d}$ Confocal images showed the cortical layer structure formation when immunostained for Ctip2 (deep cortical layer marker) and Satb2 (superficial cortical layer marker) at week 4 (c) and 12 (d), respectively. e, f Proliferation and migration of astrocytes within cerebral organoids; the differentiation pattern of astrocytes in organoids were monitored by GFAP immunostaining (astrocytic marker) at week 4 (e) and 12 (f), respectively. Scale bar: $100 \mu \mathrm{m}$.

the core portion of organoids, the immunoreactivity was measured only in the surface neuronal layers. We found the increased cleaved CASP3 immunoreactivity in the AD-E4 organoids compared to other groups with an interactive effect between APOE4 and $\mathrm{AD}$ status (Fig. 2b). Consistently, western blotting also revealed higher cleaved CASP3/CASP3 ratio in AD-E4 organoids (Fig. 2c, d). These results indicate that APOE4 and AD status synergistically exacerbate apoptosis in late stage of organoid development. Presynaptic synaptophysin and postsynaptic PSD95 were decreased in $\mathrm{AD}$ organoids groups compared to healthy subject-derived cerebral organoids, whereas no significant APOE4 effect was observed (Fig. 2c, e, and f). In contrast, on week 4, reverse transcription-quantitative PCR (RT-qPCR) revealed that cerebral organoids from $\mathrm{AD}$ patients exhibited higher mRNA levels of mature neuronal markers including MAP2, CTIP2, and $S A T B 2$, but not GFAP, an astrocyte marker (Supplementary Fig. 3A-D). Synaptophysin and PSD95 were also upregulated in AD organoid groups by week 4 (Supplementary Fig. 3E-G). While synaptophysin levels were increased in AD-E4 organoids (Supplementary Fig. 3F), APOE4 did not influence PSD95 levels (Supplementary Fig. 3G). Together, these results suggest that organoid maturation and synaptic formations are accelerated in cerebral organoids from AD patients at the early stage.

Increased $A \beta$ amounts in cerebral organoids from AD-iPSCs. To investigate the impacts of APOE4 and disease status on $A \beta$ accumulation and deposition, iPSC-derived cerebral organoids 


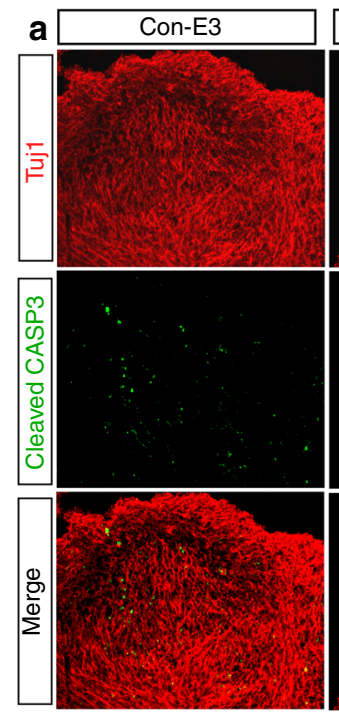

b

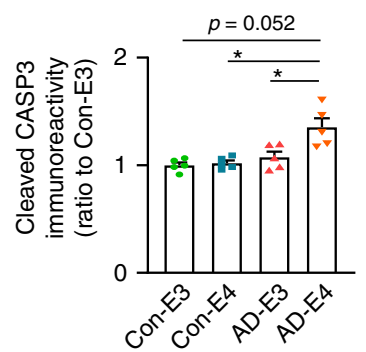

d

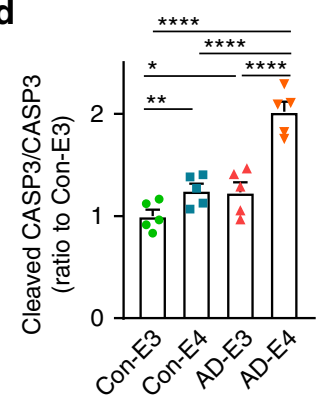

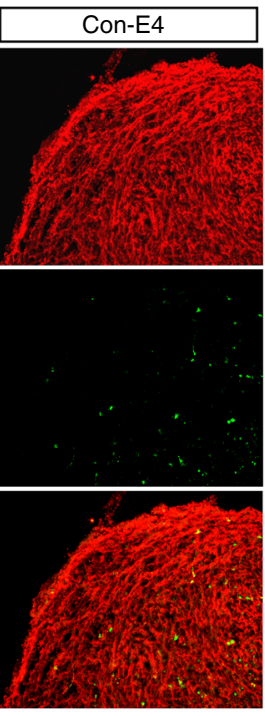
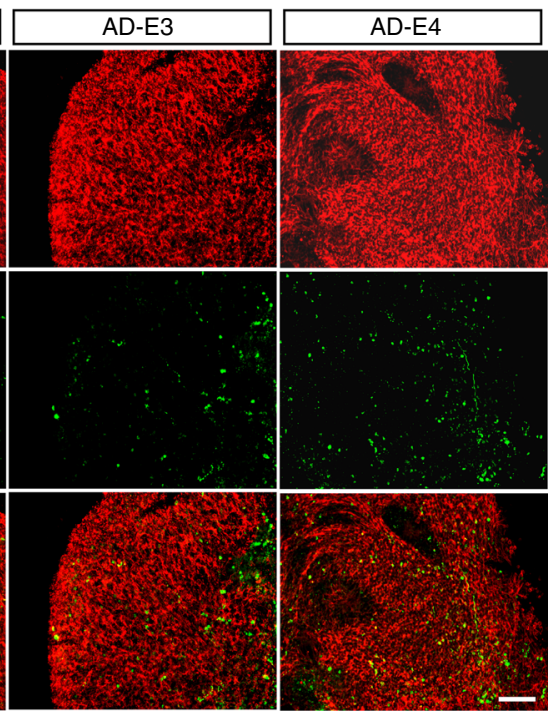

C

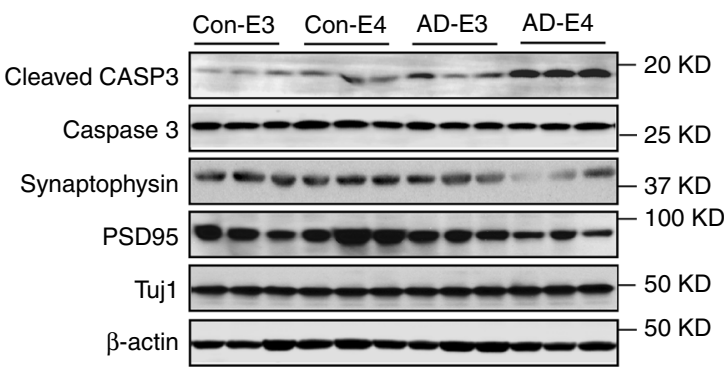

e

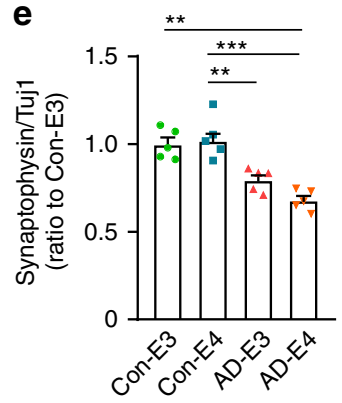

f

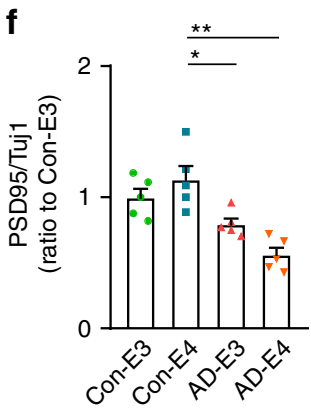

Fig. 2 APOE4 enhances apoptosis and synaptic loss in cerebral organoids from AD patients. cerebral organoids were subjected to immunostaining and western blotting at week 12. a Representative images and quantification of cellular apoptosis evaluated by immunostaining of cleaved CASP3. Scale bar: $100 \mu \mathrm{m}$. b Cleaved CASP3 immunoreactivities were quantified from 5 cerebral organoids per line, and the averaged values were compared among the groups (APOE4: $p=0.032$, AD: $p=0.0569$, APOE4 x AD: $p=0.018$, Con-E3 vs. AD-E4: $p=0.0523$, Con-E4 vs. AD-E4: $p=0.0112, A D-E 3$ vs. AD-E4: $p=$ 0.014). All data are expressed as mean \pm SEM $(N=5)$. c-f Cleaved CASP3, CASP3, synaptophysin, PSD95, and Tuj1 levels in the lysates of 4-5 cerebral organoids per line were analyzed by western blotting and quantified. All data are expressed as mean \pm SEM $(N=5)$. d Cleaved CASP3 levels were normalized to total CASP3 levels and compared among groups (APOE4: $p<0.0001$, AD: $p<0.0001$, APOE4 x AD: $p=0.0020$, Con-E3 vs. Con-E4: $p=$ 0.009, Con-E3 vs. AD-E3: $p=0.0206$, Con-E4 vs. AD-E4: $p<0.0001, A D-E 3$ vs. AD-E4: $p<0.0001$ ). Synaptophysin and PSD95 levels were normalized to Tuj1 levels and compared among groups (e APOE4: $p=0.5841, A D: p=0.0002, A P O E 4 \times A D: p=0.0453$, Con-E3 vs. AD-E4: $p=0.0069$, Con-E4 vs. ADE4: $p=0.0002$, Con-E4 vs. AD-E3: $p=0.0077$. f APOE4: $p=0.8794$, AD: $p=0.0025$, APOE4 x AD: $p=0.0551$, Con-E4 vs. AD-E3: $p=0.0404$, Con-E4 vs. AD-E4: 0.0019). ANCOVA for APOE4, AD status, and APOE4 x AD status was performed by including sex, sampling age, and source of iPSCs as co-variables, which was followed by two-sided Tukey-Kramer tests to compare between the groups with two factors (APOE4 and AD status). ${ }^{*} p<0.05$, ${ }^{\star \star} p<0.01,{ }^{\star \star \star} p<0.001,{ }^{\star \star \star \star} p<0.0001$.

were utilized for the analyses at different time points (weeks 4,8 and 12). Organoids were sequentially lysed in RIPA buffer and formic acid (FA), and subjected to the measurements for $A \beta 40$ and $A \beta 42$. Using ELISA, we detected $A \beta$ at an earlier time point than that reported by Lin et al. ${ }^{28}$, in which they assessed $A \beta$ accumulation by western blotting. Higher levels of $A \beta 40$ at week 8 (Supplementary Fig. 4A) and A $\beta 42$ at weeks 4 and 8 (Supplementary Fig. 4B) were observed in the RIPA-soluble fraction from
$\mathrm{AD}$ organoids as compared to those from healthy subject-derived cerebral organoids. The increases of $A \beta 40$ (Fig. 3a), $A \beta 42$ (Fig. $3 b$ ), and $A \beta 42 / A \beta 40$ ratio (Fig. $3 c$ ) in $A D$ organoid groups were magnified at week 12 independent of APOE4. A $\beta 40$ and $A \beta 42$ in detergent-insoluble FA fraction were undetectable at any time points. In addition, neither APOE4 nor disease status affected APP derivatives including SAPPa, sAPP $\beta$, and CTF- $\beta$ at week 12 (Fig. $3 \mathrm{~d}-\mathrm{f}$ ), whereas APOE4 but not AD status was 
a

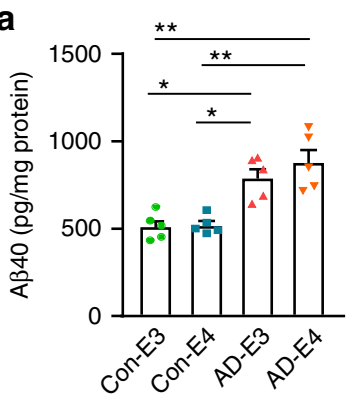

d

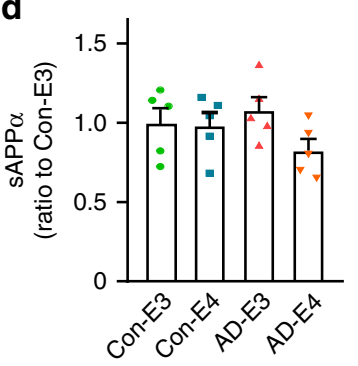

b

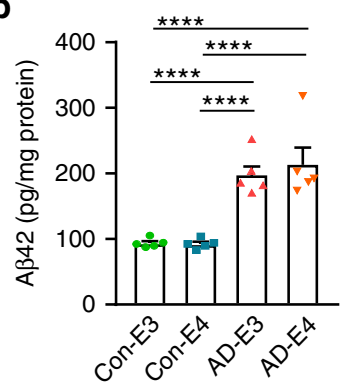

e

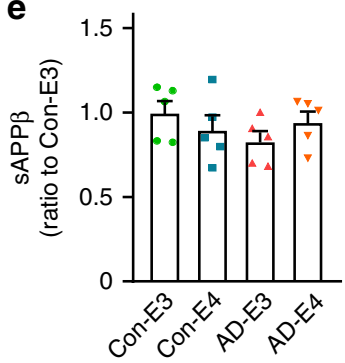

c

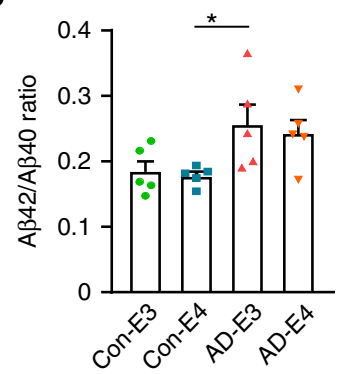

f

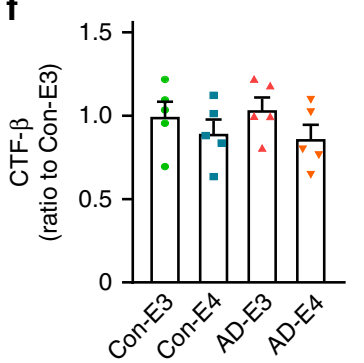

g

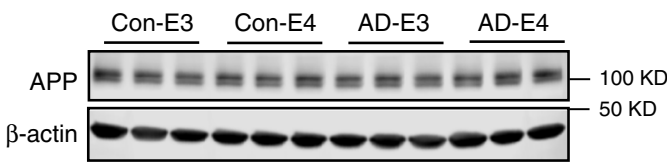

h

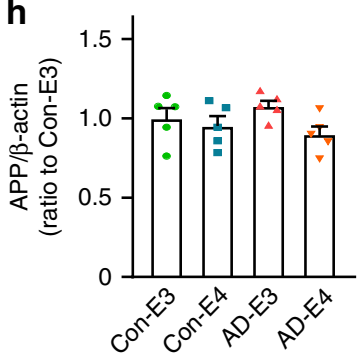

Fig. 3 Increased A $\boldsymbol{\beta}$ accumulation in iPSC-derived cerebral organoids from AD patients. Lysates of 4-5 cerebral organoids per iPSC line were analyzed by ELISA and western blotting at week 12. a-c Amounts of A $\beta 40$ (a; APOE4: $p=0.2167, \mathrm{AD}: p=0.0002$, APOE4 $\times$ AD: $p=0.4849$, Con-E3 vs. AD-E3: $p=$ 0.0287, Con-E3 vs. AD-E4: $p=0.0028$, Con-E4 vs. AD-E3: $p=0.0171$, Con-E4 vs. AD-E4: $p=0.0011)$ and A $\beta 42(\mathbf{b} ; A P O E 4: p=0.1014, A D: p<0.0001$, APOE4 x AD: $p=0.7778$, Con-E3 vs. AD-E3: $p<0.0001$, Con-E3 vs. AD-E4: $p<0.0001$, Con-E4 vs. AD-E3: $p<0.0001$, Con-E4 vs. AD-E4: $p<0.0001$ ) in the RIPA fraction were measured by ELISA. Data were normalized to the total protein concentration of the respective sample. The ratio of $A \beta 42 / A \beta 40$ was calculated accordingly (c; APOE4: $p=0.9549$, AD: $p=0.0034$, APOE4 x AD: $p=0.5331$, Con-E4 vs. AD-E3: $p=0.0397$ ). d-f Amounts of sAPP $\alpha$ (d; APOE4: $p=0.1081, \mathrm{AD}: p=0.8009$, APOE4 $\times$ AD: $p=0.1351)$, sAPP $\beta(E ; A P O E 4: p=0.7772$, AD: $p=0.4410, A P O E 4 \times$ AD: $p=0.0936)$ and CTF- $\beta$ (f; APOE4: $p=0.1150, A D: p=0.5326$, APOE4 x AD: $p=0.9877)$ in RIPA were measured by ELISA. Data are shown as ratios to Con-E3 after normalization to total protein concentration. $\mathbf{g}, \mathbf{h}$ Amounts of full-length APP were assessed by western blotting analysis using $22 \mathrm{C} 11$ monoclonal antibody in RIPA lysate (APOE4: $p=0.0331, A D: p=0.6706, A P O E 4 \times A D: p=0.3170)$. All data are expressed as mean \pm SEM $(N=5)$. ANCOVA for APOE4, AD status, and APOE4 $\times$ AD status was performed by including sex, sampling age, and source of iPSCs as co-variables, which was followed by two-sided Tukey-Kramer tests to compare between the groups with two factors (APOE4 and AD status). ${ }^{\star} p<0.05,{ }^{\star \star} p<0.01,{ }^{\star \star \star \star} p<0.0001$.

associated with lower levels of full-length APP (Fig. 3g, h). These results suggest that $A \beta$ clearance mechanism rather than APP processing is altered in cerebral organoids from $\mathrm{AD}$ patients regardless of $A P O E 4$ status, resulting in enhanced $A \beta$ accumulation and increased $A \beta 42 / A \beta 40$ ratio.

Exacerbated tauopathy in cerebral organoids from APOE4-iPSCs. To examine the tau pathology in the healthy subject-derived and $\mathrm{AD}$ patient-derived cerebral organoids carrying different $A P O E$ genotypes, we stained cerebral organoids with antiphosphorylated tau (p-tau) AT8 at week 12 and found more p-tau accumulation in organoids with APOE4 (Fig. 4a). Consistent with the results, western blotting with AT8 revealed that $A P O E 4$ and $\mathrm{AD}$ status were correlated with higher $\mathrm{p}$-tau/tau ratio in the organoids (Fig. 4b-d). When RIPA lysates (Fig. 4e) and FA fractions (Fig. $4 \mathrm{f}$ ) were subjected to ELISA, APOE4, and AD status were independently associated with p-tau upregulation in the organoids at week 12 . While RIPA-soluble p-tau increased in all groups in time, APOE4 or $\mathrm{AD}$ patient-derived organoids showed higher p-tau levels as early as week 4 (Supplementary Fig. 4C). Together, these results indicate that APOE4 and AD status aggravate tau pathology in iPSC-derived organoids without interactive effects.

When apoE levels were measured in the organoids by ELISA, higher soluble apoE levels in RIPA fraction was associated with APOE4 at week 12 (Fig. 5a); however, AD status rather than APOE4 was associated with increased insoluble apoE in the FA fraction at week 12 (Fig. 5b). Both APOE4 and AD status were associated with the increased soluble apoE levels at week 4 (Supplementary Fig. 4D). No significant differences in GFAP/ Tuj1 ratio were observed among the four groups of cerebral organoids by immunostaining (Supplementary Fig. 5A-B), RTqPCR (Supplementary Fig. 5C), and western blotting (Supplementary Fig. 5D), suggesting that $A P O E 4$ or $\mathrm{AD}$ status leads to the higher apoE level without increasing astrocyte population in the cerebral organoids. Among all groups, significant positive correlations were observed between p-tau and apoE levels in RIPA (Fig. 5c) and FA fractions (Fig. 5d). Although there were no 
a
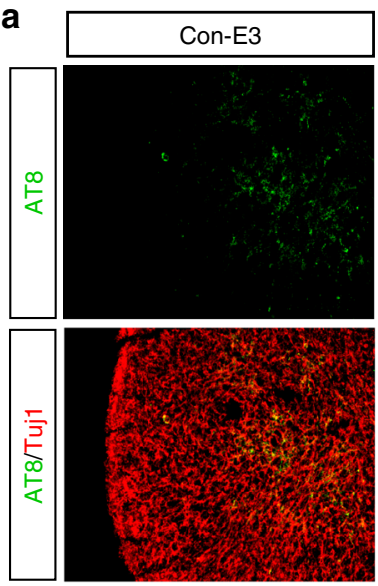

b

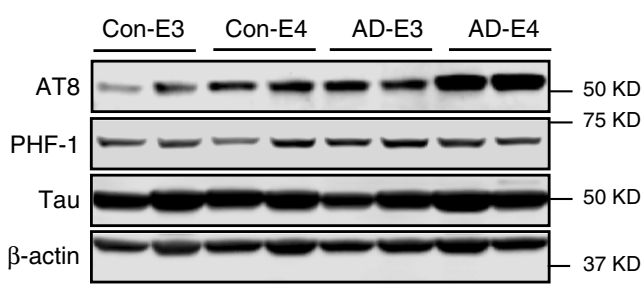

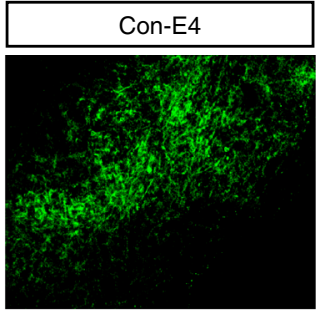

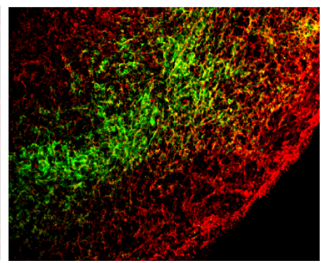

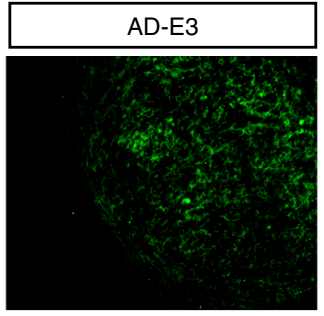

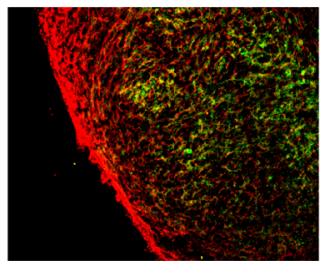

C

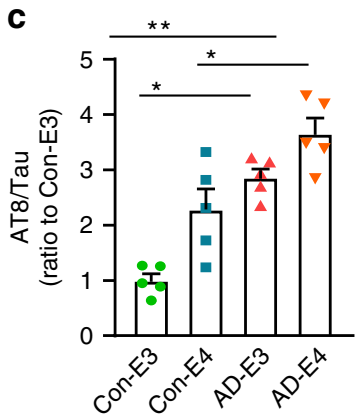

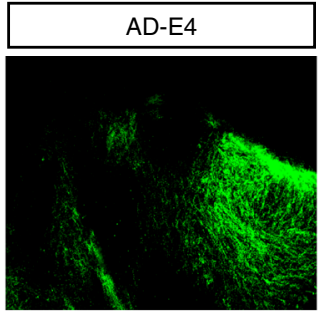

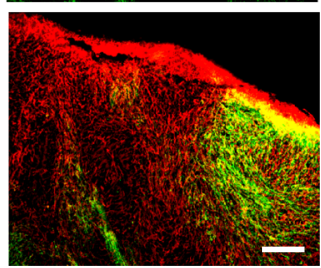

d

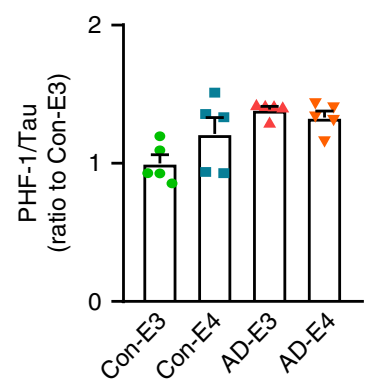

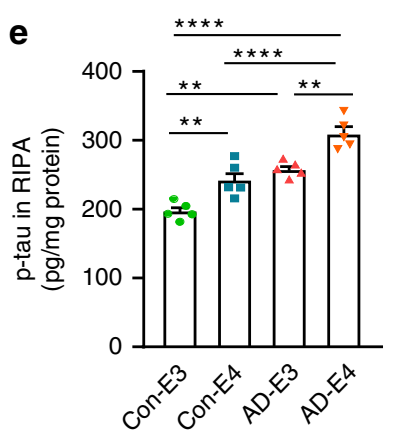

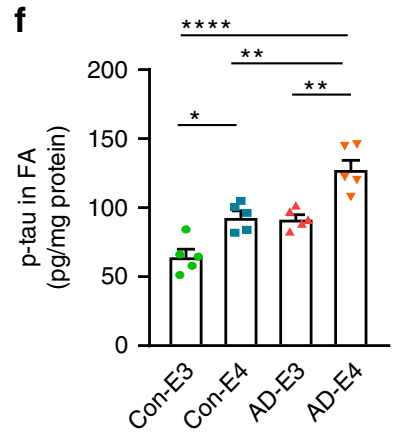

Fig. 4 APOE4 and AD status enhance p-tau levels in iPSC-derived cerebral organoids. Cerebral organoids were subjected to analyses by immunostaining, western blotting, and ELISA at week 12. a Representative images of the immunostaining for p-tau with AT8 (Ser202/Thr205) antibody. Scale bar: $100 \mu \mathrm{m}$. b-d Total tau and p-tau levels in the RIPA lysates of 4-5 cerebral organoids per line were analyzed by western blotting and quantified. The $p$-tau levels detected by AT8 antibody (c; APOE4: $p=0.0037, A D: p=0.0010, A P O E 4 \times A D p=0.6128$, Con-E3 vs. AD-E3: $p=0.0290$, Con-E3 vs. AD-E4: $p=0.0010$, Con-E4 vs. AD-E4: $p=0.0273$ ) and PHF1 (Ser396/Ser404) (d; APOE4: $p=0.3742$, AD: $p=0.0404$, APOE4 x AD: $p=0.1823$ ) were normalized to total tau. e, $\mathbf{f}$ Amounts of $p$-tau in the RIPA fraction (e; APOE4: $p<0.0001$, AD: $p<0.0001$, APOE4 x AD: $p=0.7063$, Con-E3 vs. Con-E4: $p=0.0029$, Con-E3 vs. AD-E3: $p=0.0015$, Con-E3 vs. AD-E4: $p<0.0001$, Con-E4 vs. AD-E4: $p<0.0001$, AD-E3 vs. AD-E4: $p=0.0010)$ and the FA fraction (f; APOE4: $p<0.0001, \mathrm{AD}: p=0.0005$, APOE4 x AD: $p=0.4038$, Con-E3 vs. Con-E4: $p=0.0121$, Con-E3 vs. AD-E4: $p<0.0001$, Con-E4 vs. AD$\mathrm{E} 4: p=0.0024, \mathrm{AD}-\mathrm{E} 3$ vs. AD-E4: $p=0.0012$ ) from 4 to 5 cerebral organoids per line were measured by ELISA. Data were normalized to individual total protein concentration. All data are expressed as mean \pm SEM $(N=5)$. ANCOVA for APOE4, AD status, and APOE4 $\times$ AD status was performed by including sex, sampling age, and source of iPSCs as co-variables, which was followed by two-sided Tukey-Kramer tests to compare between the groups with two factors (APOE4 and AD status). ${ }^{\star} p<0.05,{ }^{\star \star} p<0.01,{ }^{\star \star \star \star} p<0.0001$.

evident correlations between $\mathrm{A} \beta$ and apoE levels (Fig. 5e, f), $\mathrm{A} \beta$ levels (Fig. 5g, h) were also positively correlated with p-tau levels in RIPA fraction. These results suggest that $A \beta$ and apoE might associate with $\mathrm{p}$-tau through different molecular pathways.

Altered transcriptomes in cerebral organoids from AD-iPSCs. To further address the impact of APOE4 and/or AD status on transcriptional profiles in iPSC-derived organoids, we performed RNA-sequencing (RNA-seq) at week 12. We identified 1331 and 717 differentially expressed genes (DEGs) between Con-E3 and Con-E4, and between $\mathrm{AD}-\mathrm{E} 3$ and $\mathrm{AD}-\mathrm{E} 4$, respectively, with an overlap of 302 genes (Supplementary Data 1, Supplementary Fig. 5A). Among the overlapped genes, 265 genes changed in the same direction. When comparing between healthy subjectderived and $\mathrm{AD}$ patient-derived cerebral organoids, we identified 1188 DEGs between Con-E3 and AD-E3, and 501 DEGs between Con-E4 and AD-E4, with an overlap of 317 genes 304 of which were changed in the same direction (Supplementary Data 1, Supplementary Fig. 6B). Furthermore, pathway analysis revealed "Amyloid proteins" as the top-ranked network affected by the disease status and APOE4 interaction (Supplementary Fig. 6C). The top-ranked pathways enriched by DEGs were 

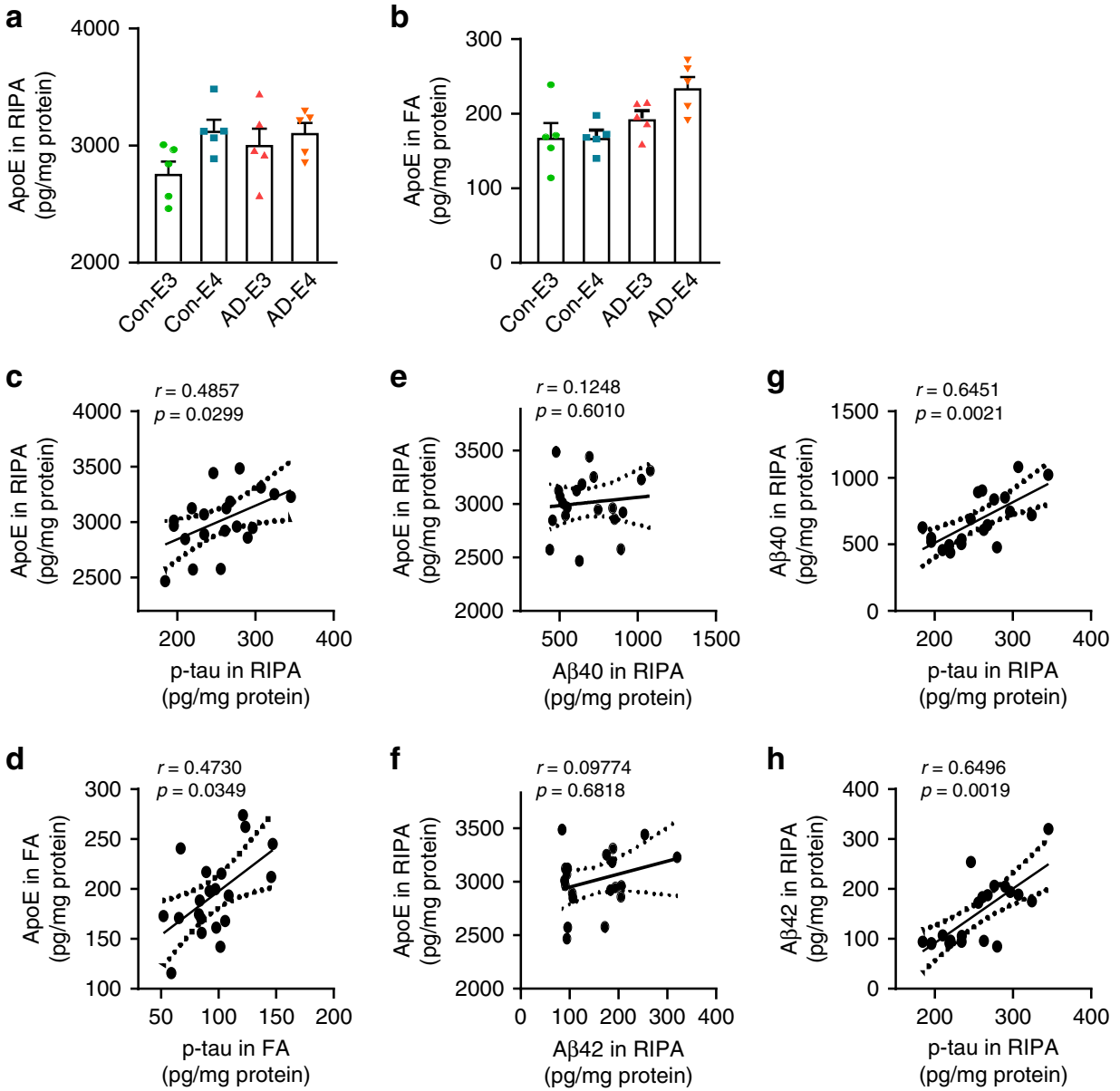

Fig. 5 Positive correlation between apoE and p-tau levels in the iPSC-derived cerebral organoids. $\mathbf{a}$, $\mathbf{b}$ Amounts of apoE in the RIPA fraction (a; APOE4: $p=0.0322$, AD: $p=0.3691$, APOE4 $\times$ AD: $p=0.4301$ ) and the FA fraction (b; APOE4: $p=0.641, \mathrm{AD}: p=0.0244, A P O E 4 \times$ AD: $p=0.4023$ ) of 4-5 cerebral organoids per line were measured by ELISA at week 12. Data were normalized to individual total protein concentration. All data are expressed as mean \pm SEM $(N=5)$. ANCOVA for APOE4, AD status, and APOE4 $\times$ AD status was performed by including sex, sampling age, and source of iPSCs as covariables, which was followed by two-sided Tukey-Kramer tests to compare between the groups with two factors (APOE4 and AD status). c-h Two-sided Spearman correlation analysis (before adjustment) of apoE vs. p-tau in RIPA (c) and FA (d) fractions, apoE vs. A $\beta 40$ (e) or A 342 (f) in RIPA fraction, and p-tau vs. $A \beta 40$ (g) or $A \beta 42(\mathbf{h})$ in RIPA fraction.

"Actin filaments" in Con-E3 vs. Con-E4 comparison (Supplementary Fig. 6D), "Regulation of epithelial-to-mesenchymal transition" in AD-E3 vs. AD-E4 comparison (Fig. S6E), "Axonal guidance" in Con-E3 vs. AD-E3 comparison (Supplementary Fig. 6F), and "Endoplasmic reticulum stress pathway" in Con-E4 vs. AD-E4 comparison (Supplementary Fig. 6G). "Innate inflammatory response" also showed up in all of the top 10ranked pathways, suggesting a potential involvement of inflammation in APOE4 effects and AD pathogenesis (Supplementary Fig. 6D-G). The expression of selected most significant changed genes (DND1, NR2F6, VPS9D1, CTH, and CDHR3) was further validated by RT-qPCR, the results of which were consistent with those from RNA-seq (Supplementary Fig. 6H-L).

To assess potential contributions of different brain cell types for these DEGs, we analyzed the RNA-seq data using CIBERSORT and CellCODE program. We found that genes detected in the cerebral organoids were mainly derived from neurons (median: 0.9231) and astrocytes (median: 0.06698), whereas oligodendrocytes compose negligible proportion of the cell population (Supplementary Fig. 7A). No significant differences in neuron and astrocyte proportion were observed among different groups (Supplementary Fig. 7B-C). As more DEGs were designated to neurons than those assigned to astrocytes in all comparisons (Supplementary Fig. 7D-G), those results suggest that neurons predominantly contributed to the phenotypes of cerebral organoids from different groups compared to astrocytes.

Weighted Gene Co-expression Network Analysis (WGCNA) identified two gene modules that were significantly associated with disease status: module magenta was positively correlated with $\mathrm{AD}$, while module yellow was positively correlated with healthy subject-derived cerebral organoids (negatively correlated with AD) (Fig. 6a). Genes in the yellow module were enriched for RNA metabolism dysregulation (Fig. 6b), which included ERCC4, DGCR8, POLR3A, CLP1, HSPA4, PNO1, VPS18, RAD17, LCMT2, and RPUSD2 as top-ranked hub genes (Fig. 6c). We further validated expressions of selected genes by RT-qPCR and western blotting, and confirmed decreased mRNA and protein levels in AD-E4 organoids (Fig. $6 \mathrm{~d}, \mathrm{f}, \mathrm{j}-\mathrm{l}$ ), Genes in the magenta module were enriched for those related to DNA and nucleosome metabolism pathways (Supplementary Fig. 8A). CHIC1, H2BC7, H2BC8, ASB3, H2BC18, H2BC21, H13, ARRDC3, and TBL1XR1-AS1 were identified as top-ranked hub genes (Supplementary Fig. 8B). We further confirmed the increased mRNA levels of CHIC1, ASB3, and ARRDC3 by RTqPCR in AD organoids, in particular those with APOE4 (Supplementary Fig. 8C-E). Since some of the hub genes in the yellow module (PNO1, DGCR8, VPS18, and HSPA4) are 


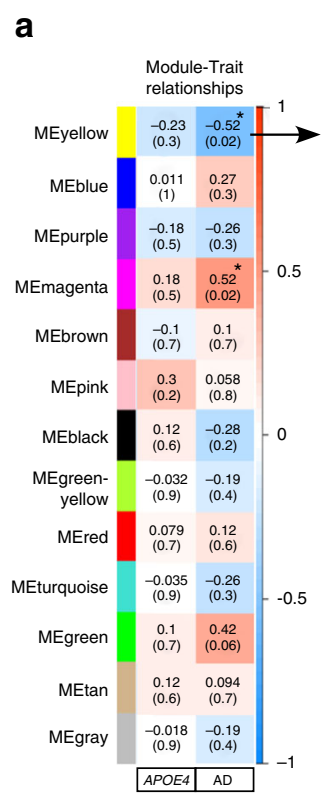

b

Gene ontologies enriched in the module

"ncRNA metabolic process *ncRNA processing *tRNA metabolic process

${ }^{*}$ tRNA processing

"Organic cyclic compound binding

"RNA modic compound binding

"RNA binding

RNA binding

bounded membrane-
RNA processing

c

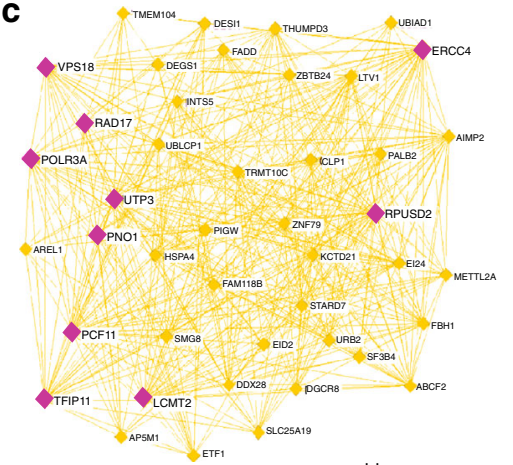

d

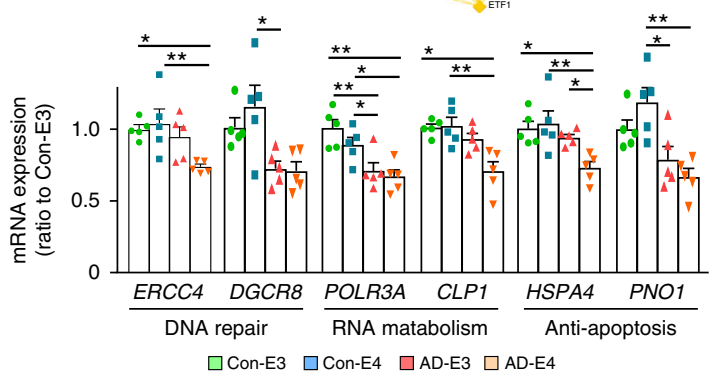

e

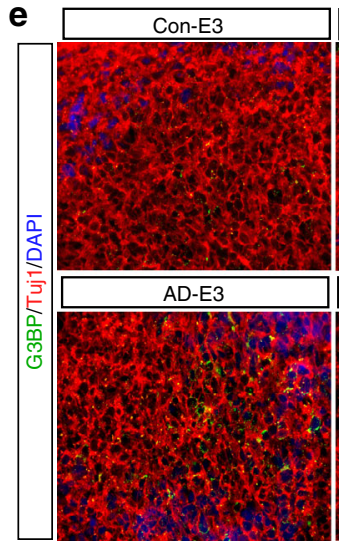

g
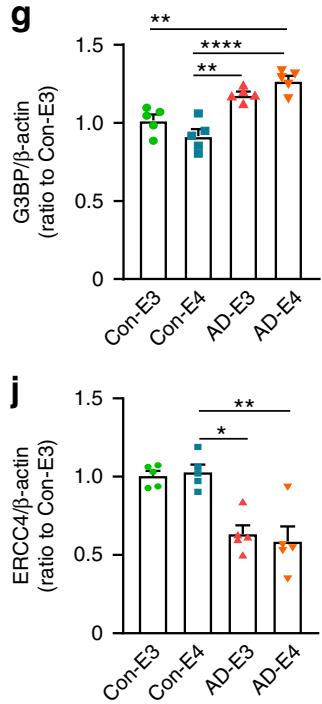

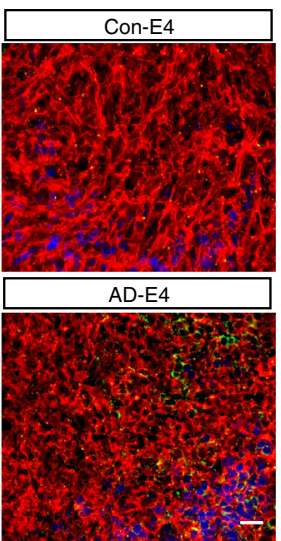

h

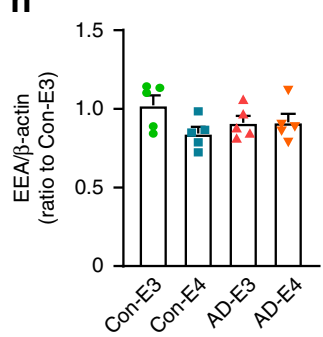

k

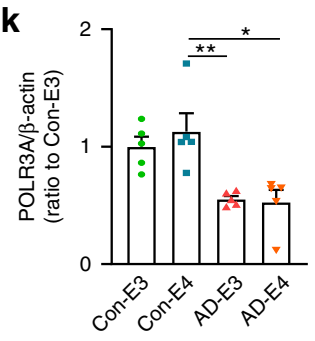

i
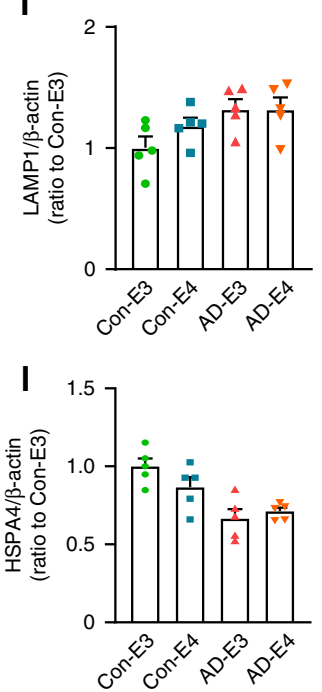

closely related to lysosomal stress granules formation under cellular stress conditions ${ }^{29,30}$, we immunostained the cerebral organoids for a stress granule marker Ras GTPase-activating protein-binding protein 1 (G3BP) in the cerebral organoids at week 12, and found an increase of G3BP-positive punctates in
AD organoids (Fig. 6e). We further quantified G3BP, endosome marker protein early endosome antigen 1 (EEA1), and lysosome marker protein lysosomal-associated membrane protein 1 (LAMP1) by western blotting, and found the interactive effect between APOE4 and AD status; G3BP 
Fig. 6 Transcriptomics profiling of the iPSC-derived cerebral organoids by WGCNA. a Module-trait relationships between modules and APOE genotype, and between modules and AD status are shown. b, $\mathbf{c}$ Top gene ontologies and interaction of genes enriched in the yellow module genes. Purple nodes are hub genes (top 10 highest connectivity). d The mRNA expressions of ERCC4 (APOE4: $p=0.0576, \mathrm{AD}: p=0.0064, A P O E 4 \times$ AD: $p=0.0742$, Con-E3 vs. AD-E4: $p=0.0164$, Con-E4 vs. AD-E4: $p=0.0049$ ), DGCR8 (APOE4: $p=0.1088, A D: p=0.0469, A P O E 4 \times$ AD: $p=0.3298$, Con-E4 vs. AD-E3: $p=$ 0.0409), POLR3A (APOE4: $p=0.3926, A D: p=0.0004, A P O E 4 \times$ AD: $p=0.1349$, Con-E3 vs. AD-E3: $p=0.0048$, Con-E3 vs. AD-E4: $p=0.0084$, Con-E4: AD-E3: $p=0.0221$, Con-E4 vs. AD-E4: $p=0.0484), C L P 1$ (APOE4: $p=0.0953, A D: p=0.0039, A P O E 4 \times A D: p=0.1831$, Con-E3 vs. AD-E4: $p=0.0157$, Con-E4 vs. AD-E4: $p=0.0066$ ), HSPA4 (APOE4: $p=0.0501, \mathrm{AD}: p=0.0077, \mathrm{APOE4} \times \mathrm{AD}: p=0.0410$, Con-E3 vs. AD-E4: $p=0.0171, \mathrm{Con}-\mathrm{E} 4$ vs. AD-E4: $p=0.0038, A D-E 3$ vs. AD-E4: $p=0.0339$ ), and PNO1 (APOE4: $p=0.8087, A D: p=0.0022, A P O E 4 \times A D: p=0.1706$, Con-E4 vs. AD-E3: $p=0.0218$, Con-E4 vs. AD-E4: $p=0.0038$ ) were quantified by RT-qPCR. e Representative images of the immunostaining for G3BP and Tuj1. Scale bar: $50 \mu \mathrm{m}$. f-i G3BP, EEA1, and LAMP1 levels were analyzed by western blotting. The levels of G3BP (g; APOE4: $p=0.6345$, AD: $p<0.0001, A P O E 4 \times$ AD: $p=0.0133$, Con-E3 vs. AD-E4: $p=0.0025$, Con-E4 vs. AD-E3: $p=0.0019$, Con-E4 vs. AD-E4: $p<0.0001$ ), EEA1 (h; APOE4: $p=0.2792, A D: p=0.6789, A P O E 4 \times$ AD: $p=0.0671$ ), and LAMP1 (I; APOE4: $p=0.2558, A D: p=0.0954, A P O E 4 \times A D: p=0.5646)$ were normalized to $\beta$-actin. $\mathbf{j}-\mathbf{I}$ ERCC4, POLR3A, and HSPA4 were analyzed by western blotting. The levels of ERCC4 (j; APOE4: $p=0.9762$, AD: $p=0.0016$, APOE4 $\times$ AD: $p=0.3378$, Con-E4 vs. AD-E3: $p=0.0241$, Con-E4 vs. AD-E4: $p=0.0054)$, POLR3A (k; APOE4: $p=0.2095, A D: p=0.0018$, APOE4 x AD: $p=0.7764$, Con-E4 vs. AD-E3: $p=0.0048$, Con-E4 vs. AD-E4: $p=0.0149$ ), and HSPA4 (l; APOE4: $p=0.6526$, AD: $p=0.0152$, APOE4 x AD: $p=0.2371$ ) were normalized to $\beta$-actin. All data are expressed as mean \pm SEM $(N=5)$. ANCOVA for APOE4, AD status, and APOE4 x AD status was performed by including sex, sampling age, and source of iPSCs as co-variables, which was followed by two-sided Tukey-Kramer tests to compare between the groups with two factors (APOE4 and AD status). ${ }^{*} p<0.05$, ${ }^{\star \star} p<0.01,{ }^{\star \star \star \star} p<0.0001$.

significantly increased in organoids from $\mathrm{AD}$ patients carrying APOE4 (Fig. 6f, g). No significant changes were observed in EEA1 (Fig. 6h) and LAMP1 levels (Fig. 6i). These results imply that the disruption of RNA metabolism in cerebral organoids from $\mathrm{AD}$ patients accelerates stress granule formation especially in the presence of APOE4.

Conversion of $A P O E 4$ to $A P O E 3$ attenuates $\mathrm{AD}$ pathologies. Using a parental iPSC line with $A P O E \varepsilon 4 / \varepsilon 4$ from a sporadic $\mathrm{AD}$ patient (Par-E4/4) and the isogenic iPSC line with $A P O E$ $\varepsilon 3 / \varepsilon 3$ (Iso-E3/3) ${ }^{19}$, we tested whether converting APOE4 to $A P O E 3$ could ameliorate APOE4-related phenotypes in iPSCderived organoids. While no obvious size difference was observed between cerebral organoids from the parental line and isogenic line at week 12 (Fig. 7a), immunostaining showed the reduction of cleaved CASP 3 immunoreactivity in the edge of cerebral organoids from Iso-E3/3 compared to Par-E4/4 organoids (Fig. 7b, Supplementary Fig. 10C). The levels of cleaved CASP3 in the center of the cerebral organoids at week 12 were measured to evaluate the possible influence of necrotic core. No significant differences in the center of cerebral organoids from the parental line and isogenic line were observed (Supplementary Fig. 10B). Correlation analysis showed no significant correlation between the levels of cleaved CASP3 at the center with those at the edge, suggesting the apoptosis at the edge of cerebral organoids is not driven by the necrotic core at the center (Supplementary Fig. 10D). Western blotting also showed a lower cleaved CASP3/CASP3 ratio in Iso-E3/3 organoids than Par-E4/4 organoids (Fig. 7c). In addition, Iso-E3/3 organoids showed the decreased $A \beta 40$ (Fig. $7 \mathrm{~d}$ ) and $\mathrm{A} \beta 42$ (Fig. 7e) levels in the RIPA lysate, although the $A \beta 42 / 40$ ratio (Fig. 7f) remained unaffected by APOE4 conversion. Whereas RIPAsoluble apoE levels were not affected (Fig. $7 \mathrm{~g}$ ), insoluble apoE in FA fraction was decreased in the Iso-E3/3 organoids (Fig. $7 \mathrm{~h}$ ). Immunostaining (Fig. 7i) and western blotting (Fig. 7j) showed that $A P O E 4$ conversion to APOE3 reduced p-tau levels and $\mathrm{p}$ tau/tau ratios in both RIPA (Fig. 7k) and FA fractions (Fig. 7l) in Iso-E3/3 organoids compared with Par-E4/4 organoids, which were confirmed by ELISA. Converting APOE4 to APOE3 reduced the levels of G3BP in cerebral organoids when analyzed by western blotting (Fig. $7 \mathrm{~m}$ ). Taken together, these results indicate that APOE4-related phenotypes observed in $\mathrm{AD}$ patient-derived organoids can largely be reversed through genome editing to APOE3.

\section{Discussion}

While $\mathrm{AD}$ is neuropathologically diagnosed through postmortem assessments in patients with dementia ${ }^{31}$, recent research framework has established antemortem $\mathrm{AD}$ classification using biomarkers for $A \beta$ deposition (A), pathologic tau (T), and neurodegeneration $(\mathrm{N})^{32}$. Thus, to explore the complex $\mathrm{AD}$ pathogenesis and develop therapeutic interventions for the disease, the establishment of human models system recapitulating ATN phenotypes has become essential. Toward this, we have comprehensively investigated $\mathrm{AD}$-related pathogenic pathways using iPSC-derived 3-D cerebral organoids from sporadic AD patients with or without APOE4. cerebral organoids not only simulate intrinsic spatial patterning, but also display acquisition of cell identity in a timed manner that closely mimics the temporal patterning with sequential neuronal layer formation, accompanied with matured astrocytes ${ }^{21,22,33}$. Furthermore, the phenotypes related to aberrant extracellular protein aggregation can be recapitulated only in $3-\mathrm{D}$ culture systems ${ }^{28,34,35}$. Using iPSC-derived cerebral organoid system, we identified multiple APOE4- and/or AD disease status-dependent pathogenic pathways, revealing complex etiology associated with APOE4, the strongest genetic risk factor for late-onset $\mathrm{AD}$.

Consistent with results from 2-D cultures of iPSC-derived neurons ${ }^{13,18,28,36}$, we found the elevated $A \beta 40$ and $A \beta 42$ levels in RIPA-soluble fraction in AD patient-derived organoids regardless of APOE4 status. Since APP processing was not altered, AD brain organoids may have compromised machineries for enzymatic $A \beta$ degradation and/or cellular $A \beta$ clearance. Of note, a significant reduction of soluble $A \beta$ levels was observed in the cerebral organoids from a sporadic $\mathrm{AD}$ patient upon isogenic conversion of APOE4 to APOE3. Thus, it is possible that APOE4 and other gene variants synergistically facilitate $\mathrm{A} \beta$ accumulation in $\mathrm{AD}$ organoids. Notably, insoluble $A \beta$ and amyloid plaque pathology was undetectable by ELISA and immunostaining in our organoid models from sporadic $\mathrm{AD}$ patients at week 12 . While $\mathrm{A} \beta$ plaque deposition is detected 2-4 months after the differentiation in iPSC-organoid models from familial $\mathrm{AD}$ and Down syndrome patients $^{35,37}$, those from sporadic $\mathrm{AD}$ patients likely have $\mathrm{A} \beta$ deposition 6 months after differentiation ${ }^{28}$. Thus, longer differentiation duration may be required to assess amyloid deposition in iPSC-derived organoids from sporadic AD cases.

Another pathological hallmark of AD is the abnormal phosphorylation, mislocalization, and aggregation of $\operatorname{tau}^{38,39}$, although tauopathy is also detected in non-AD neurodegenerative diseases including frontotemporal dementia (FTD) and progressive 
a

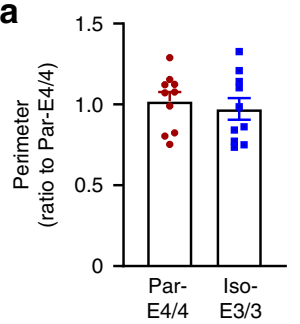

d
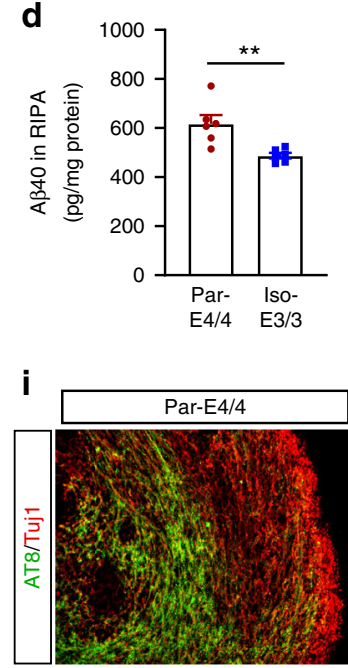

k

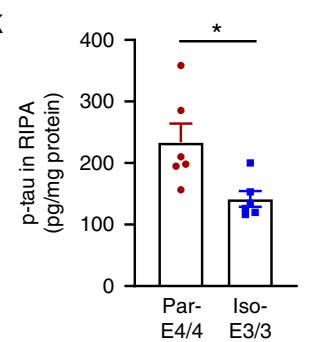

b

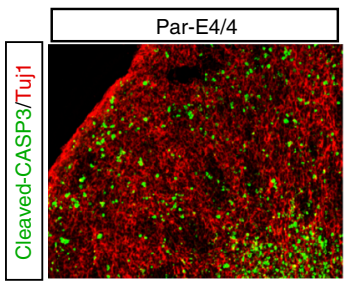

e
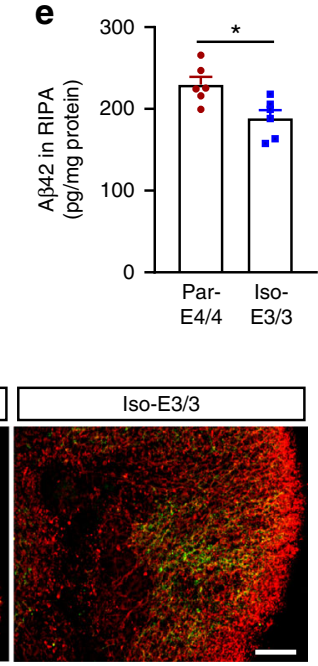

I

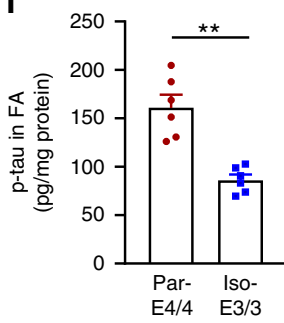

C
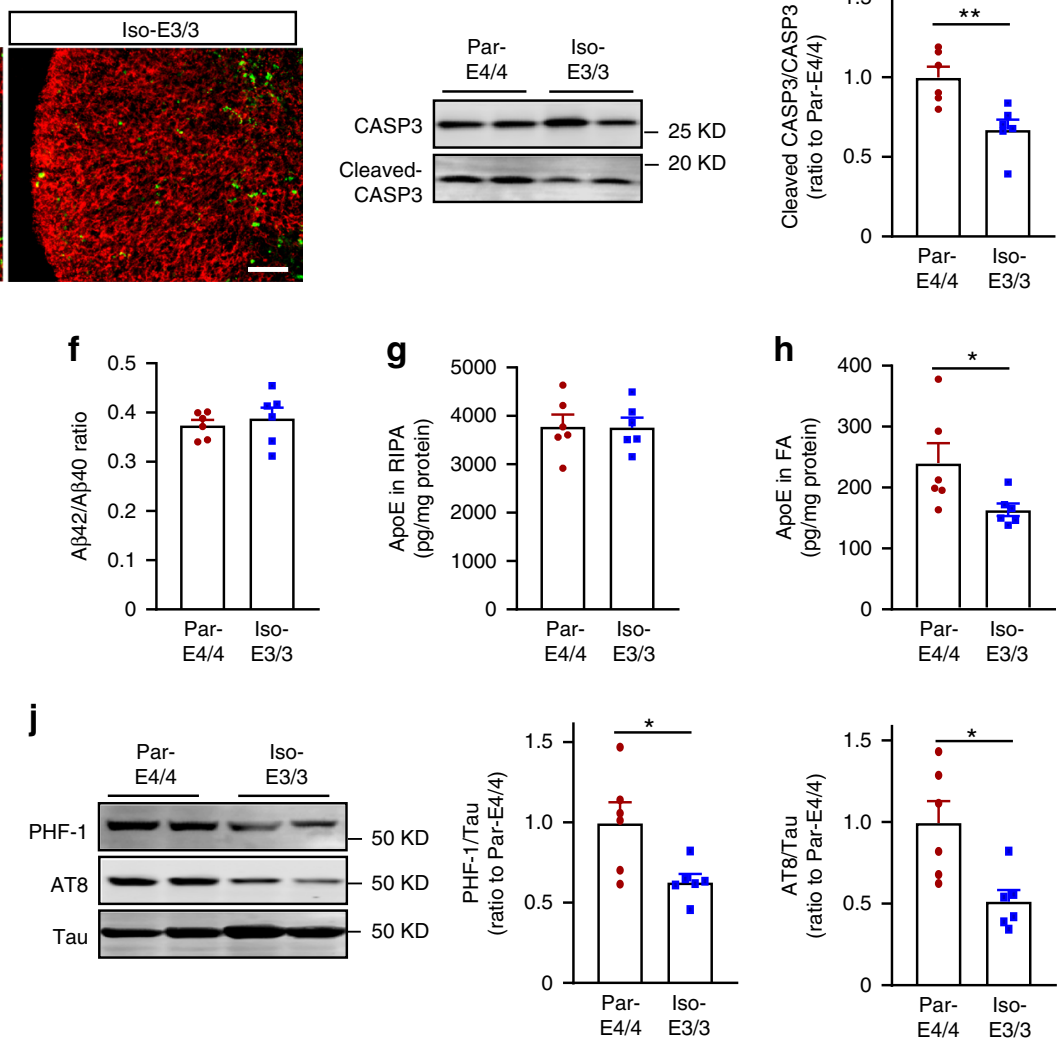

m

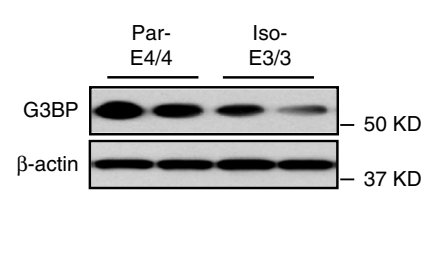

Fig. 7 Isogenic conversion of APOE4 to APOE3 attenuates AD-related phenotypes in cerebral organoids. The iPSC-derived cerebral organoids from an $\mathrm{AD}$ patient carrying $A P O E \varepsilon 4 / \varepsilon 4$ (Par-E4/4) and the $A P O E \varepsilon 3 / \varepsilon 3$ isogenic line (Iso-E3/3) were analyzed at week 12. a The perimeters of cerebral organoids were measured $(n=10)$. b Representative images of cellular apoptosis with the immunostaining for cleaved CASP3. Scale bar: $100 \mu \mathrm{m}$. c Cleaved CASP3 and CASP3 levels in the lysates were analyzed by western blotting and quantified $(p=0.0043)$. $\mathbf{d}-\mathbf{f}$ Amounts of $A \beta 40(\mathbf{d}: p=0.0043)$ and $A \beta 42$ (e: $p=$ $0.0152)$ in RIPA fraction were measured by ELISA. Data were normalized to individual protein concentrations. The ratio of $A \beta 42 / A \beta 40$ (f) was calculated accordingly. $\mathbf{g}$, $\mathbf{h}$ Amounts of apoE in RIPA ( $\mathbf{g})$ and FA (h: $p=0.0411)$ were measured by ELISA. Data were normalized to individual total protein concentrations. i Representative images of the immunostaining for p-tau with AT8 antibody. Scale bar: $100 \mu \mathrm{m}$. $\mathbf{j}$ Total tau and p-tau in RIPA lysates were analyzed by western blotting and quantified. The $p$-tau detected by AT8 antibody and PHF1 antibody were normalized to total tau (AT8: $p=0.0152$, PHF1: $p=0.0411)$. $\mathbf{k}$, I Amounts of $p$-tau in RIPA (k: $p=0.0152)$ and FA (I: $p=0.0022)$ were measured by ELISA. Data were normalized to individual total protein concentration. $\mathbf{m}$ G3BP, levels in RIPA lysates were analyzed by western blotting and quantified. The levels of G3BP were normalized to $\beta$-actin $(p=0.0022)$. $\mathbf{c}-\mathbf{h}, \mathbf{j}-\mathbf{m}$ Lysates of 4-5 cerebral organoids were analyzed as one sample. All data are expressed as mean \pm SEM $(n=6)$. Two-sided Mann-Whitney $U$ tests were performed to determined statistical significance, ${ }^{\star} p<0.05,{ }^{\star}{ }^{\star} p<0.01$.

supranuclear palsy $(\mathrm{PSP})^{40}$. Indeed, tau phosphorylation is accelerated in iPSC-derived neurons from $\mathrm{AD}$ patients ${ }^{13,41}$. Furthermore, APOE4 has been shown to exacerbate AD-related tau pathologies in cell and mouse models as well as human patients ${ }^{10,42}$. Our results also found that $\mathrm{AD}$ status and APOE4 are independently associated with the increased levels of p-tau in iPSC-derived cerebral organoids. Given that neither APOE4 nor apoE amounts influenced $A \beta$ levels in the iPSC-derived cerebral organoids, APOE4 may accelerate the p-tau accumulation in an $\mathrm{A} \beta$-independent manner. Of note, apoE and p-tau levels show positive correlations in both RIPA and FA fractions, which is consistent with the results from mouse studies showing that Apoe deficiency ameliorates tauopathy ${ }^{42}$. In addition, Wang et al. has shown that p-tau levels were increased in APOE-deficient iPSCderived neurons when treated with lysates of iPSC-derived neurons with $A P O E 4^{19}$. Thus, although further studies are necessary, APOE4 likely induces a gain-of-toxic effect on $\mathrm{p}$-tau accumulation in iPSC-derived cerebral organoids.

Intriguingly, we found marked increase in cellular apoptosis and reductions of synaptic proteins in mature organoids from $\mathrm{AD}$ patients compared to healthy subject-derived cerebral organoids. In supporting our findings, iPSC-derived neurons from a sporadic $\mathrm{AD}$ patient have been reported for their hyper-excitable calcium signaling phenotype, increased cytotoxicity and apoptosis, and 
reduced neurite length in comparison to those from healthy individuals ${ }^{36}$. Wang et al. also found expression of apoE4 was associated with increased GABAergic neuron degeneration ${ }^{19}$. More supportively, the gain-of-toxic-function of apoE4 could be ameliorated when apoE4 was converted to apoE3 by gene editing, which is highly consistent with what we found in the cerebral organoid model ${ }^{19}$. In contrast, at the early neuronal differentiation stage, increased mature neuronal markers and synaptic proteins were detected in AD-derived organoids. Given that similar observations were reported in iPSC-derived neurons from $\mathrm{AD}$ subjects ${ }^{43}$, specific factors facilitating excess neuronal maturation in the early stage may be commonly preserved in iPSCs from AD patients, resulting in enhanced neurodegeneration in the late stage. While APOE4 likely leads to early maturation of neurons ${ }^{28}$ partially consistent with our results, we demonstrated that APOE4 further exacerbates the effects of $\mathrm{AD}$ status on apoptosis induction and synaptic loss in cerebral organoids at week 12 . Thus, it is tempting to speculate that accelerated neuronal differentiation/maturation is synergistically induced by AD-related factors and APOE4 in the presymptomatic stage, and that the mechanistic exhaustion followed by neuronal dysfunction may contribute to disease development, although further studies are needed.

Our transcriptomic analysis demonstrated that an enrichment of gene sets involving RNA metabolisms is predominantly affected by $\mathrm{AD}$ status in the iPSC-derived cerebral organoids; while APOE4 did not have a strong effect on the profiles. RNA metabolism process is highly dynamic and requires a complex interplay among RNA-binding proteins (RBPs) ${ }^{44}$. Thus, the disruption of RBP homeostasis is frequently involved in the pathology and genetics of neurodegenerative diseases including FTD and amyotrophic lateral sclerosis (ALS), where aggregationprone RBPs often co-localize with stress granules ${ }^{44-46}$. Interestingly, we also found a significant increase in stress granules in organoids from $\mathrm{AD}$ patients. While various RBPs have been shown to deposit in tau inclusions ${ }^{47-49}$, internalized tau sensitizes cells to stress by promoting formation and stability of stress granules ${ }^{49}$. Thus, enhanced $p$-tau accumulation may trigger transcriptional changes and stress granule formation in organoids from AD patients. There were also reductions of mRNAs in genes related to DNA repair (ERCC4 and DGCR8) $)^{50,51}$ and antiapoptosis (PNO1 and HSPA4) ${ }^{52,53}$ in the organoids from $\mathrm{AD}$ patients. However, further studies are necessary to determine if those are causatively or consequently involved in neurodegeneration.

Although our results demonstrate that iPSC-derived cerebral organoid is a useful modeling system to investigate AD- and APOE4-related phenotypes, we should state several limitations. First, the core of cerebral organoids shows necrosis-like changes after week 12, likely due to the lack of vascular systems maintaining proper supplies of nutrition and gas exchange as well as immune cells to eliminate cell debris and toxic molecules. To address this caveat, emerging technology has further developed cerebral organoid models in which vascular cells ${ }^{19,54}$ or microglia are incorporated $28,55,56$. Thus, in future studies, we plan to utilize the iPSC-derived cerebral organoid system with neuroimmune and/or neurovascular system to establish a more advanced platform for AD research. Second, there was heterogeneity in organoid size and growth rate even though identical iPSC lines were used. We also noticed that maturation of astrocytes and the detection of $A \beta$ or apoE in cerebral organoids may vary depending on differentiation protocols ${ }^{28,33,57}$. For example, Lin et al. ${ }^{28}$ found lower soluble apoE levels in cerebral organoids with APOE4 compared to those with APOE3, which is different from our results. The differences in the medium used for differentiation and the time points of medium change may potentially contribute to the different results under the stress conditions induced by APOE4 or AD status. The technical differences and the intrinsic variability using iPSC-derived cerebral organoid models should be considered whenever comparing results from different studies. Third, because of the limited availability of patient-derived iPSC lines, we could not match sex among our study groups in our study. Nonetheless, we did adjust sex as a variable in our analysis. Further optimization and standardization in the iPSC-derived cerebral organoid modeling system should be able to overcome these weaknesses.

In conclusion, our study established a true 3-D human cerebral organoid system to address $\mathrm{AD}$ pathogenesis. We successfully recapitulated AD-related pathologies related to ATN classification in our model system. APOE4 predominantly aggravates $\mathrm{p}$-tau accumulation, while AD status is associated with higher levels of $\mathrm{A} \beta$ and p-tau, apoptosis, synaptic loss and increased stress granule formation. Importantly, APOE4 synergistically accelerates apoptosis and stress granule formation with $\mathrm{AD}$ status in our $3 \mathrm{D}$ model. Thus, exploring molecular mechanisms in the interaction between APOE4- and AD-related pathways should provide clues as to how APOE4 vastly increases $\mathrm{AD}$ risk. Because isogenic conversion of APOE4 into APOE3 reverses much of the ADrelated phenotypes in cerebral organoids from $\mathrm{AD}, A P O E 4$ might be a promising therapeutic target for $\mathrm{AD}$.

\section{Methods}

Generation of iPSCs from human skin fibroblasts. Human skin biopsies from normal individuals and AD patients with $A P O E \varepsilon 3 / \varepsilon 3$ or $\varepsilon 4 / \varepsilon 4$ genotype were obtained from Mayo Clinic patients under IRB protocols with patient consent for research, which was approved by the Mayo Clinic Institutional Review Board. $A P O E$ genotype was confirmed by Sanger sequencing using DNA samples from fibroblast lines. Cells were cultured in fibroblast medium containing $10 \%$ fetal bovine serum (FBS) (Gemini Bio-Products). The iPSCs were generated by electroporation of three episomal vectors into the fibroblasts using the NHDF nucleofector kit (Lonza) ${ }^{23,58}$. Three micrograms of expression plasmid mixtures were electroporated into $6 \times 10^{5}$ fibroblasts with $100 \mu \mathrm{l}$ transfection reagents. After transfection, fibroblasts were plated onto a $100 \mathrm{~mm}$ Matrigel (Corning) coated dish After 5 days of culture, the fibroblast medium was replaced with TeSR-E7 complete medium (Stemcell Technologies) and changed every day. IPSC colonies were isolated and expanded after 3-4 weeks in culture. The iPSC colonies were passaged using Dispase (Stemcell Technologies) and subjected to rock inhibitor Y27632 (Sigma-Aldrich) treatment for the first $24 \mathrm{~h}$.

Trilineage differentiation of human iPSCs. Three germs layer differentiation was used to confirm the pluripotency of iPSCs using the STEMdiff Trilineage Differentiation kit (Stemcell Technologies) according to manufacturer's instructions with some modification ${ }^{59}$. When cells were $\sim 70 \%$ confluent, iPSCs were passaged using Accutase (Stemcell Technologies) and plated into an AggreWell ${ }^{\mathrm{m}} 800$ plate (Stemcell Technologies) to form embryonic bodies (EB) in mTeSR1 medium for 2 days. EBs were washed out from the AggreWell plate and transferred to 6-well non-tissue culture treated plates (Corning) in specific differentiation mediums for each lineage. EBs were subjected to differentiation into mesoderm and endoderm lineages for 5 days or an ectoderm lineage for 7 days, then seeded onto Matrigelcoated plates for further analysis. Differentiation was assessed by immunostaining for germ layer-specific markers (Endoderm: SOX17; Mesoderm: Brachyury; Ectoderm: Nestin/Sox2).

Cerebral organoid culture. Cerebral organoids were generated according to manufacturer's instructions of the commercial STEMdiff ${ }^{\mathrm{m}}$ Cerebral Organoid Kit (Stemcell Technologies). On day 0, any pre-differentiated cells in the hiPSCs culture were removed by scraping under a microscope. Human iPSC colonies were dissociated into single-cell suspension with Accutase. In total, 9000 cells were then plated into each well of a U-bottom ultra-low-attachment 96-well plate in EB formation media (medium A) supplemented with $10 \mu \mathrm{M}$ Y27632. An extra $100 \mu \mathrm{l}$ EB medium was added on day 2 and day 4, respectively. EBs were moved to 24-well low attachment plates in neural induction medium (medium B) for another 3-5 days. EBs were further embedded into $20 \mu \mathrm{l}$ of matrigel and cultured in neural expansion medium (medium $\mathrm{C}+\mathrm{D}$ ) for 3 days in 6-well low attachment plates for organoid formation. In the final stage, the organoids were transferred to $10-\mathrm{cm}$ dishes with neural culture medium (medium E), and moved to an orbital shaker for further culture. Medium E was replaced with neuronal maturation medium after 4 weeks, which was composed of DMEM/F12 + Neurobasal Medium (1:1) supplemented with N2, B27, BDNF $(20 \mathrm{ng} / \mathrm{ml})$, GDNF $(20 \mathrm{ng} / \mathrm{ml})$, ascorbic acid $(200 \mu \mathrm{M})$, and dbcAMP $(100 \mathrm{nM})$ (Sigma-Aldrich) ${ }^{60}$. Cerebral organoids were 
collected at different time points for immunostaining and other biochemical analysis. We differentiated 20 lines of iPSCs into cerebral organoids within a close time frame, and used cerebral organoids with normal size range (typically $2-4 \mathrm{~mm}$ of diameter) for our experiments.

Immunostaining. At pre-defined time points, cerebral organoids were fixed in $4 \%$ paraformaldehyde for $30 \mathrm{~min}$ then washed with PBS three times. After fixation, organoids were dehydrated with $30 \%$ sucrose in PBS at $4^{\circ} \mathrm{C}$. Organoids were then embedded with optical cutting temperature (OCT) compound (VWR) and frozen on dry ice. Frozen tissue was sectioned at $30 \mu \mathrm{m}$ using a cryostat and collected on ultra-frosted glass microscope slides. Sections were stored at $-20^{\circ} \mathrm{C}$. For immunostaining, sections were permeabilized in $0.25 \%$ Triton X-100 and blocked with blocking buffer containing $4 \%$ normal donkey serum, $2 \%$ BSA, and $1 \mathrm{M}$ glycine in PBS. Sections were then incubated with primary antibodies in blocking buffer overnight at $4{ }^{\circ} \mathrm{C}$. The information of primary antibodies and their dilutions used in this study are as follows: Nanog (Cell Signaling, 4903, 1:300), TRA-1-60 (Abcam, ab16288, 1:300), SSEA4 (Abcam, ab16287, 1:300), Sox17 (Abcam, ab84990, 1:300), Brachyury (R\&D, AF2085, 1:300), Nestin (Abcam, ab18102, 1:500), Sox2 (Abcam, ab97959, 1:500), TUJ1 (Abcam, ab78078, 1:1000), TUJ1 (Sigma, T2200, 1:1000), CTIP2 (Abcam, ab18465, 1:100), SATB2 (Abcam, ab34735, 1:100), GFAP (Millipore, MAB360, 1:300), cleaved Caspase-3 (Cell Signaling Technology, 9579, 1:300), AT8 (Thermo Fisher Scientific, MN1020, 1:300), and G3BP (BD, 611126, 1:300). After washing three times with PBS, samples were incubated with fluorescently conjugated secondary antibodies (Alexa Fluor 488 and 594 conjugates, Invitrogen, 1:500) for $2 \mathrm{~h}$ at room temperature and washed three times with PBS before mounting with the glass coverslip. Fluorescent signals were detected by fluorescence microscopy (model IX71 Invert, Olympus), confocal laser scanning fluorescent microscopy (model LSM510 Invert, Carl Zeiss), and Keyence fluorescence microscopy (model BZ-X, Keyence). The fluorescence signal was quantified using ImageJ software.

Tissue processing. Cerebral organoids were harvested at pre-defined time points and lysed with RIPA Lysis and Extraction Buffer supplemented with Protease and Phosphatase Inhibitor Cocktails for Cell Lysis (Roche). Lysed samples were sonicated and incubated for $60 \mathrm{~min}$ on ice. Samples were centrifuged in an ultracentrifuge (Beckman-Coulter) at $100,000 \times g$, for $1 \mathrm{~h}$ at $4^{\circ} \mathrm{C}$. Supernatants (soluble fraction) were collected and the pellet was resuspended in $50 \mu \mathrm{l}$ of $70 \%$ formic acid (FA), sonicated, and neutralized with $2.5 \mathrm{M}$ Tris buffer ( $\mathrm{pH}$ 8.5) (insoluble fraction). Total protein concentration in the soluble fraction was determined using a Pierce BCA Protein Assay Kit.

Western blotting. Samples in soluble fraction were loaded into a $4-20 \%$ sodium dodecyl sulfate-polyacrylamide gel (Bio-Rad), and transferred to PVDF Immobilon FL membranes (Millipore). After blocking with $5 \%$ non-fat milk in PBS, membranes were blotted overnight with primary antibodies in $5 \%$ non-fat milk containing $0.01 \%$ Tween-20, and then probed with LI-COR IRDye secondary antibodies or horseradish peroxidase-conjugated secondary antibody, detected by SuperSignal West Femto Chemiluminescent Substrate (Pierce). The information of primary antibodies and their dilutions used in this study are as follows: Cleaved Caspase-3 (Cell Signaling Technology, 9661, 1:1000), Caspase-3 (Cell Signaling Technology, 9662, 1:1000), PSD95 (Abcam, ab2723, 1:1000), Synaptophysin (Abcam, ab8049, 1:1000), AT8 (Thermo Fisher Scientific, MN1020, 1:1000), PHF1 (Abcam, ab184951, 1:1000), Tau5 (Millipore, 577801, 1:1000), APP (Thermo Fisher Scientific, 14-9749-82, 1:1000), GFAP (Millipore, MAB360, 1:1000), EEA1 (Cell Signaling Technology, 2411, 1:1000), LAMP1 (Cell Signaling Technology, 9091, 1:1000), G3BP (BD, 611126, 1:1000), ERCC4 (Fitzgerald, 10R-4026, 1:1000), POLR3A (Abcam, ab96328, 1:1000), and HSPA4 (Cell Signaling Technology, 3303, 1:1000). All uncropped blot images with molecular size markers were available in the Source data file.

ELISA quantification. The A $\beta 40, \mathrm{~A} \beta 42$, sAPP $\alpha$, sAPP $\beta, \mathrm{CTF}-\beta$, and phospho-tau levels in both RIPA and formic acid fractions were measured using the Human $\beta$ Amyloid (1-40) ELISA Kit (Thermo Fisher, KHB3481), Human $\beta$-Amyloid (1-42) ELISA Kit (Thermo Fisher, KHB3441), Human sAPPa Assay Kit (IBL, 27734), Human sAPP $\beta$ Assay Kit (IBL, 27732), Human CTF- $\beta$ ELISA kit (IBL, 27776), and the Tau (Phospho) [pS396] Human ELISA Kit (Thermo Fisher, KHB7031) according to the manufacturers' instructions. For human apoE ELISA, 96-well plates were coated overnight with an apoE antibody (WUE4) in carbonate buffer at $4{ }^{\circ} \mathrm{C}$. The plates were blocked with $1 \%$ milk in PBS, and washed three times with PBS. Recombinant apoE3 and apoE4 (Fitzgerald) were used as standards for the ELISA. Samples were diluted and incubated at $4{ }^{\circ} \mathrm{C}$ overnight. The plates were washed and incubated with biotin-conjugated goat anti-apoE antibody (Meridian Life Science) for $2 \mathrm{~h}$ at room temperature. After incubation with Horseradish Peroxidase Avidin D (Vector Laboratories) for $90 \mathrm{~min}$ at room temperature, the plate was developed by adding tetramethylbenzidine Super Slow substrate (Sigma). The reaction was stopped and read at $450 \mathrm{~nm}$ with a microplate reader (Biotek). Results were normalized to total protein concentration of the cell lysate.
RT-qPCR. RNA was extracted via the Trizol/chloroform method, followed by DNase and cleanup using the RNase-Free DNase Set and the RNeasy Mini Kit (QIAGEN). The quantity and quality of all RNA samples for RNA-seq was determined by the Agilent 2100 Bioanalyzer using the Agilent RNA 6000 Nano Chip (Agilent Technologies, CA). The cDNA was prepared with the iScript cDNA synthesis kit (Bio-Rad). Real-time qPCR was conducted with Universal SYBR Green Supermix (Bio-Rad) using an iCycler thermocycler (Bio-Rad). The 2exp $(-\Delta \Delta \mathrm{Ct})$ method was used to determine the relative expression of each gene with $A C T B$ gene coding $\beta$-actin as a reference. The primers used to amplify target genes by RT-qPCR are as follows: ACTB F (5'-CTGGCACCACACCTTCTACAATG-3') and R ( $5^{\prime}$-AATGTCACGCACGATTTCCCGC-3'), MAP2 F (5'-CAGGTGGCGG ACGTGTGAAAATTGAGAGTG- $3^{\prime}$ ) and R (5'-CACGCTGGA TCTGCCTGGGG ACTGTG-3'), CTIP2 F (5'-GTTGTGCAAATGTAGCTGGAA- ${ }^{\prime}$ ) and R (5'-GAA GATGACCACCTGCTCTC- $\left.3^{\prime}\right)$, SATB2 F (5'-CCTTACGCAGAATCTCAGACA A- $\left.3^{\prime}\right)$ and R (5'-CCAGATATCTACCAGCAAGTCAG-3'), GFAP F (5'-CTGTT GCCAGAGATG GAGGTT-3') and R (5'-TCATCGCTCAGGAGGTCCTT-3'), ERCC4 F (5'-TTTGTGAGG AAACTGTATCTG TGG-3') and R (5'-GTCTGT ATAGCAAGCATGGTAGG-3'), DGCR8 (PPH17455A, QIAGEN), POLR3A F (5'-TCTGGAGACCTGTAGGGACAA-3') and R (5'-CTGGCTCACCAATGCTC T-3'), CLP1 (PPH10372A, QIAGEN), HSPA4 F (5'-GCAACA GCAGCAGACAC CAGC-3') and R (5'-GCCTTCTTGGCTTGGGGTGGT-3'), PNO1 (PPH15310A, QIAGEN), DND1 F (5'-CTCCACAGGCACCCTGAATG-3') and R (5'-GGTGCC ATAGGTCCCTGTCC- $3^{\prime}$ ), NR2F6 F (5'-TTTAGTCGATTTCACGCGGAC-3') and R (5'-ATCTGTTCAGACGGGTACTCG-3'), VPS9D1 F (5'-CTCAGACGTCC CCAGGAA CTG-3') and R (5'-GCCAGACAAGGACAGCTCGTTC- $\left.3^{\prime}\right)$, CTH F (5'-GAAGACCTACT GGAAGAT- $3^{\prime}$ ) and R (5'-GGAATACTAGCTGTGACT- $3^{\prime}$ ), CDHR3 F (5'-TGTGAAGGA TGAGGTTGGTG- $3^{\prime}$ ) and R (5'-TCCAGGGTTTG CTCTTTCTAC-3'), CHIC1 (PPH09017A, QIAGEN), ASB3 F (5'-CATACTTAT TTCATCGGGTGC- $3^{\prime}$ ) and R (5'-GGTAACTGCCA ACTGTCCTC-3'), ARRDC3 F (5'-ATCCCAGTGTGATGTGACGA-3') and R (5'-TTTGCAA CAGAATCGG AAAA- $\left.3^{\prime}\right)$, TUBB3 F (5'-CCTCCGTGTAGTGACCCTT-3') and R (5'-GGCCTT TGGACATCTCTTCAG-3').

RNA-seq, quality control, and normalization. Twenty mRNA samples were sequenced at Mayo Clinic using Illumina HiSeq 4000. Reads were mapped to the human reference genome hg38. Raw gene read counts and sequencing quality control were generated using the Mayo Clinic RNA-Seq analytic pipeline: MAPRSeq Version $3.0^{61}$. Conditional quantile normalization (CQN) was performed on the raw gene counts to correct for gene length differences, GC bias, global technical variations, and to obtain similar quantile-by-quantile distributions of gene expression levels across samples ${ }^{62}$. Based on the bi-modal distribution of the CQNnormalized and log2-transformed reads per $\mathrm{kb}$ per million (RPKM) gene expression values, genes with an average of $\log 2 \mathrm{RPKM}>=3$ in at least one group were considered expressed. Using this selection threshold, 18,291 genes were included in the downstream analysis.

Differential gene expression and pathway analysis. Differential gene expression analyses were performed using the Partek Genomics Suite (Partek Inc., St. Louis, $\mathrm{MO}$ ). Gene expression between AD patient- and healthy subject-derived cerebral organoids, between $A P O E$ genotypes, and the interaction between $\mathrm{AD}$ disease status and $A P O E$ genotype were calculated using analyses of variance models (ANOVA). Pathway analyses of differentially expressed genes, defined by $p<0.05$ and $\mid$ fold change $\mid \geq 1.5$, were performed using MetaCore pathway analysis (๔ MetaCore (Feb 2020) of Clarivate Analytics. All rights reserved).

DEGs cell-type distribution analysis of RNA-seq data. Cell proportion was estimated using marker genes described in BRETIGEA ${ }^{63}$, a published reference dataset, and the CIBERSORT program ${ }^{64}$. First, the top 50 marker genes of neuron, astrocyte, and oligodendrocyte cells were obtained from BRETIGEA (version 1.0.0) ${ }^{63}$, among which 30 neuronal markers, 27 astrocytic markers, and 19 oligodendrocyte markers are also present in both reference dataset and our iPSC dataset. Next, expression levels of these markers in sorted neurons, astrocytes, and oligodendrocytes were obtained from the reference dataset of Zhang et al. ${ }^{65}$. Finally, CIBERSORT analytical tool (version 1.0.1) (64 $^{6}$ was applied to estimate cellular composition in the iPSC dataset using the selected maker gene expression in reference dataset and in the iPSC dataset for neurons, astrocytes, and oligodendrocytes. To estimate in which celltype genes might be regulated, we applied CellCODE R package ${ }^{66}$ to assess the interaction between group variable and surrogate proportion variables of each cell type. Because oligodendrocytes compose negligible proportion of the cell population in all but one sample, oligodendrocyte was not considered in the following analysis. Specifically, getAllSPV function in CellCODE was applied to refine the top 50 markers of neuron and astrocyte obtained from BRETIGEA, and to obtain surrogate variables of neuron and astrocyte populations using the remaining markers through singular value decomposition. Next, cellPopT function was applied to calculate the $t$ statistics of the interaction term between group and the surrogate variables. Cell type with the largest $t$ statistics is the designated cell type.

WGCNA. To identify groups of genes that are correlated with $\mathrm{AD}$ disease status or with $A P O E$ genotype, we performed weighted gene co-expression network analysis 
(WGCNA $)^{67}$ using the log2-transformed, CQN-normalized gene expression values. We used the soft power of 16 , hybrid dynamic tree cutting, a minimum module size of 50 genes, and a minimum height for merging modules at 0.3 to build a signed hybrid co-expression networks. Each gene module was summarized by the first principal component of the scaled module expression profiles (module eigengene). Each module was assigned a unique color identifier, and genes that did not fulfill these criteria for any of the modules were assigned to the gray module. To assess the correlation of modules to $\mathrm{AD}$ disease status and $A P O E$ genotype, we defined healthy subject-derived cerebral organoids as 0 and $A D$ patient-derived cerebral organoids as 1 ; and defined the APOE3 genotype as 0 , and the APOE4 genotype as 1 . Modules were annotated using R package anRichment (https:// horvath.genetics.ucla.edu/html/CoexpressionNetwork/GeneAnnotation/). The connection among the top hub genes in the yellow module was visualized using VisANT $^{68}$.

Statistical analyses and reproducibility. ANCOVA for APOE4, AD status, sex, sampling age and source of iPSCs was performed to determine the interaction effect between APOE4 and AD status on each continuous variable for western blotting, ELISA, or RT-qPCR from four groups of cerebral organoids, followed by Tukey-Kramer tests to compare between selected two groups using JMP software version 15.0. For batch difference and isogenic cerebral organoids comparison, the Mann-Whitney $U$ test was performed to determine the significance using GraphPad Prism version 8.0. Spearman correlation analysis was used to analyze the association between proteins using GraphPad Prism. Experiments were repeated in two independent differentiation batches. Data were presented as mean \pm SEM. A $p$ value of $<0.05$ was considered statistically significant.

Reporting summary. Further information on research design is available in the Nature Research Reporting Summary linked to this article.

\section{Data availability}

Full scans of the gels and blots are available in Source data file. All relevant data are available from the corresponding author upon reasonable request. All the other data supporting the findings of this study are available within the article and its supplementary information files and from the corresponding author upon reasonable request. A reporting summary for this article is available as a Supplementary Information file. The RNA-seq data are available via the AD Knowledge Portal [https:// adknowledgeportal.synapse.org]. The AD Knowledge Portal is a platform for accessing data, analyses, and tools generated by the Accelerating Medicines Partnership (AMPAD) Target Discovery Program and other National Institute on Aging (NIA)-supported programs to enable open-science practices and accelerate translational learning. The data, analyses, and tools are shared early in the research cycle without a publication embargo on secondary use. Data are available for general research use according to the following requirements for data access and data attribution [https://adknowledgeportal.synapse. org/DataAccess/Instructions]. For access to content described in this manuscript see [https://doi.org/10.7303/syn22307008]. Source data are provided with this paper.

Received: 27 December 2019; Accepted: 2 October 2020;

Published online: 02 November 2020

\section{References}

1. Selkoe, D. J. Toward a remembrance of things past: deciphering Alzheimer disease. Harvey Lect. 99, 23-45 (2003).

2. Jack, C. R. Jr. et al. Hypothetical model of dynamic biomarkers of the Alzheimer's pathological cascade. Lancet Neurol. 9, 119-128 (2010).

3. DeTure, M. A. \& Dickson, D. W. The neuropathological diagnosis of Alzheimer's disease. Mol. Neurodegener. 14, 32 (2019).

4. Corder, E. H. et al. Gene dose of apolipoprotein E type 4 allele and the risk of Alzheimer's disease in late onset families. Science 261, 921-923 (1993).

5. Kim, J., Basak, J. M. \& Holtzman, D. M. The role of apolipoprotein E in Alzheimer's disease. Neuron 63, 287-303 (2009).

6. Liu, C. C., Liu, C. C., Kanekiyo, T., Xu, H. \& Bu, G. Apolipoprotein E and Alzheimer disease: risk, mechanisms and therapy. Nat. Rev. Neurol. 9, 106-118 (2013).

7. Yu, J. T., Tan, L. \& Hardy, J. Apolipoprotein E in Alzheimer's disease: an update. Annu Rev. Neurosci. 37, 79-100 (2014).

8. Najm, R., Jones, E. A. \& Huang, Y. Apolipoprotein E4, inhibitory network dysfunction, and Alzheimer's disease. Mol. Neurodegener. 14, 24 (2019).

9. Huynh, T. V., Davis, A. A., Ulrich, J. D., Holtzman, D. M. \& Apolipoprotein, E. and Alzheimer's disease: the influence of apolipoprotein $\mathrm{E}$ on amyloid-beta and other amyloidogenic proteins. J. Lipid Res. 58, 824-836 (2017).

10. Huang, Y. \& Mucke, L. Alzheimer mechanisms and therapeutic strategies. Cell 148, 1204-1222 (2012).
11. Golde, T. E., Schneider, L. S. \& Koo, E. H. Anti-abeta therapeutics in Alzheimer's disease: the need for a paradigm shift. Neuron 69, 203-213 (2011).

12. Ashe, K. H. \& Zahs, K. R. Probing the biology of Alzheimer's disease in mice. Neuron 66, 631-645 (2010)

13. Israel, M. A. et al. Probing sporadic and familial Alzheimer's disease using induced pluripotent stem cells. Nature 482, 216-220 (2012).

14. Yagi, T. et al. Modeling familial Alzheimer's disease with induced pluripotent stem cells. Hum. Mol. Genet. 20, 4530-4539 (2011).

15. Kondo, T. et al. Modeling Alzheimer's disease with iPSCs reveals stress phenotypes associated with intracellular Abeta and differential drug responsiveness. Cell Stem Cell 12, 487-496 (2013).

16. Liu, Q. et al. Effect of potent gamma-secretase modulator in human neurons derived from multiple presenilin 1-induced pluripotent stem cell mutant carriers. JAMA Neurol. 71, 1481-1489 (2014).

17. Centeno, E. G. Z., Cimarosti, H. \& Bithell, A. 2D versus 3D human induced pluripotent stem cell-derived cultures for neurodegenerative disease modelling. Mol. Neurodegener. 13, 27 (2018).

18. Muratore, C. R. et al. The familial Alzheimer's disease APPV717I mutation alters APP processing and Tau expression in iPSC-derived neurons. Hum. Mol. Genet. 23, 3523-3536 (2014).

19. Wang, C. et al. Gain of toxic apolipoprotein E4 effects in human iPSC-derived neurons is ameliorated by a small-molecule structure corrector. Nat. Med. 24, 647-657 (2018).

20. Lancaster, M. A. et al. Cerebral organoids model human brain development and microcephaly. Nature 501, 373-379 (2013).

21. Lancaster, M. A. \& Knoblich, J. A. Generation of cerebral organoids from human pluripotent stem cells. Nat. Protoc. 9, 2329-2340 (2014).

22. Renner, M. et al. Self-organized developmental patterning and differentiation in cerebral organoids. EMBO J. 36, 1316-1329 (2017).

23. Okita, K. et al. A more efficient method to generate integration-free human iPS cells. Nat. Methods 8, 409-412 (2011).

24. Zhao, J. et al. APOE epsilon4/epsilon4 diminishes neurotrophic function of human iPSC-derived astrocytes. Hum. Mol. Genet. 26, 2690-2700 (2017).

25. Brookhouser, N., Zhang, P., Caselli, R., Kim, J. J. \& Brafman, D. A. Generation and characterization of two human induced pluripotent stem cell (hiPSC) lines homozygous for the Apolipoprotein e4 (APOE4) risk variantAlzheimer's disease (ASUi005-A) and healthy non-demented control (ASUi006-A). Stem Cell Res. 32, 145-149 (2018).

26. Brookhouser, N., Zhang, P., Caselli, R., Kim, J. J. \& Brafman, D. A. Generation and characterization of human induced pluripotent stem cell (hiPSC) lines from an Alzheimer's disease (ASUi001-A) and non-demented control (ASUi002-A) patient homozygous for the Apolipoprotein e4 (APOE4) risk variant. Stem Cell Res. 24, 160-163 (2017).

27. Yamazaki, Y. et al. Apolipoprotein E and Alzheimer disease: pathobiology and targeting strategies. Nat. Rev. Neurol. 15, 501-518 (2019).

28. Lin, Y. T. et al. APOE4 causes widespread molecular and cellular alterations associated with Alzheimer's disease phenotypes in human iPSC-derived brain cell types. Neuron 98, 1141-1154.e1147 (2018).

29. Niewidok, B. et al. Single-molecule imaging reveals dynamic biphasic partition of RNA-binding proteins in stress granules. J. Cell Biol. 217, 1303-1318 (2018).

30. Van Treeck, B. et al. RNA self-assembly contributes to stress granule formation and defining the stress granule transcriptome. Proc. Natl Acad. Sci. USA 115, 2734-2739 (2018).

31. Gaugler, J. et al. 2019 Alzheimer's disease facts and figures. Alzheimer's \& Dementia 15, 321-387 (2019).

32. Jack, C. R. Jr. et al. NIA-AA Research Framework: toward a biological definition of Alzheimer's disease. Alzheimers Dement 14, 535-562 (2018).

33. Quadrato, G. et al. Cell diversity and network dynamics in photosensitive human brain organoids. Nature 545, 48-53 (2017).

34. Choi, S. H. et al. A three-dimensional human neural cell culture model of Alzheimer's disease. Nature 515, 274-278 (2014).

35. Gonzalez, C. et al. Modeling amyloid beta and tau pathology in human cerebral organoids. Mol. Psychiatry 23, 2363-2374 (2018).

36. Balez, R. et al. Neuroprotective effects of apigenin against inflammation, neuronal excitability and apoptosis in an induced pluripotent stem cell model of Alzheimer's disease. Sci. Rep. 6, 31450 (2016).

37. Raja, W. K. et al. Self-organizing $3 \mathrm{D}$ human neural tissue derived from induced pluripotent stem cells recapitulate Alzheimer's disease phenotypes. PLoS ONE 11, e0161969 (2016).

38. Alonso, A. C., Zaidi, T., Grundke-Iqbal, I. \& Iqbal, K. Role of abnormally phosphorylated tau in the breakdown of microtubules in Alzheimer disease. Proc. Natl Acad. Sci. USA 91, 5562-5566 (1994).

39. Mi, K. \& Johnson, G. V. The role of tau phosphorylation in the pathogenesis of Alzheimer's disease. Curr. Alzheimer Res 3, 449-463 (2006)

40. Meeter, L. H., Kaat, L. D., Rohrer, J. D. \& van Swieten, J. C. Imaging and fluid biomarkers in frontotemporal dementia. Nat. Rev. Neurol. 13, 406-419 (2017). 
41. Ochalek, A. et al. Neurons derived from sporadic Alzheimer's disease iPSCs reveal elevated TAU hyperphosphorylation, increased amyloid levels, and GSK3B activation. Alzheimers Res. Ther. 9, 90 (2017).

42. Shi, Y. et al. ApoE4 markedly exacerbates tau-mediated neurodegeneration in a mouse model of tauopathy. Nature 549, 523-527 (2017).

43. Meyer, K. et al. REST and neural gene network dysregulation in iPSC models of Alzheimer's disease. Cell Rep. 26, 1112-1127.e1119 (2019).

44. Nussbacher, J. K., Tabet, R., Yeo, G. W. \& Lagier-Tourenne, C. Disruption of RNA metabolism in neurological diseases and emerging therapeutic interventions. Neuron 102, 294-320 (2019).

45. Liu, E. Y., Cali, C. P. \& Lee, E. B. RNA metabolism in neurodegenerative disease. Dis. Model Mech. 10, 509-518 (2017).

46. Johnson, E. C. B. et al. Deep proteomic network analysis of Alzheimer's disease brain reveals alterations in RNA binding proteins and RNA splicing associated with disease. Mol. Neurodegener. 13, 52 (2018).

47. Vanderweyde, T. et al. Contrasting pathology of the stress granule proteins TIA-1 and G3BP in tauopathies. J. Neurosci. 32, 8270-8283 (2012).

48. Maziuk, B. F. et al. RNA binding proteins co-localize with small tau inclusions in tauopathy. Acta Neuropathol. Commun. 6, 71 (2018).

49. Brunello, C. A., Yan, X. \& Huttunen, H. J. Internalized Tau sensitizes cells to stress by promoting formation and stability of stress granules. Sci. Rep. 6 30498 (2016).

50. Brookman, K. W. et al. ERCC4 (XPF) encodes a human nucleotide excision repair protein with eukaryotic recombination homologs. Mol. Cell Biol. 16, 6553-6562 (1996).

51. Calses, P. C. et al. DGCR8 mediates repair of UV-induced DNA damage independently of RNA processing. Cell Rep. 19, 162-174 (2017).

52. Shen, A. et al. EBF1-mediated upregulation of ribosome assembly factor PNO1 contributes to cancer progression by negatively regulating the p53 signaling pathway. Cancer Res. 79, 2257-2270 (2019).

53. Zuo, D., Subjeck, J. \& Wang, X. Y. Unfolding the role of large heat shock proteins: new insights and therapeutic implications. Front. Immunol. 7, 75 (2016).

54. Mansour, A. A. et al. An in vivo model of functional and vascularized human brain organoids. Nat. Biotechnol. 36, 432-441 (2018).

55. Abreu, C. M. et al. Microglia increase inflammatory responses in iPSC-derived human brainspheres. Front. Microbiol. 9, 2766 (2018).

56. Ormel, P. R. et al. Microglia innately develop within cerebral organoids. Nat. Commun. 9, 4167 (2018).

57. Linkous, A. et al. Modeling patient-derived glioblastoma with cerebral organoids. Cell Rep. 26, 3203-3211.e3205 (2019).

58. Wren, M. C. et al. Frontotemporal dementia-associated N279K tau mutant disrupts subcellular vesicle trafficking and induces cellular stress in iPSCderived neural stem cells. Mol. Neurodegener. 10, 46 (2015).

59. Awe, J. P. et al. Putative immunogenicity expression profiling using human pluripotent stem cells and derivatives. Stem Cells Transl. Med. 4, 136-145 (2015).

60. Nimsanor, N. et al. Induced pluripotent stem cells (iPSCs) derived from a symptomatic carrier of a S305I mutation in the microtubule-associated protein tau (MAPT)-gene causing frontotemporal dementia. Stem Cell Res. 17, 564-567 (2016).

61. Kalari, K. R. et al. MAP-RSeq: Mayo analysis pipeline for RNA sequencing. BMC Bioinforma. 15, 224 (2014).

62. Hansen, K. D., Irizarry, R. A. \& Wu, Z. Removing technical variability in RNA-seq data using conditional quantile normalization. Biostatistics 13, 204-216 (2012).

63. McKenzie, A. T. et al. Brain cell type specific gene expression and coexpression network architectures. Sci. Rep. 8, 8868 (2018).

64. Newman, A. M. et al. Robust enumeration of cell subsets from tissue expression profiles. Nat. Methods 12, 453-457 (2015).

65. Zhang, Y. et al. Purification and characterization of progenitor and mature human astrocytes reveals transcriptional and functional differences with mouse. Neuron 89, 37-53 (2016).
66. Chikina, M., Zaslavsky, E. \& Sealfon, S. C. CellCODE: a robust latent variable approach to differential expression analysis for heterogeneous cell populations. Bioinformatics 31, 1584-1591 (2015).

67. Langfelder, P. \& Horvath, S. WGCNA: an R package for weighted correlation network analysis. BMC Bioinforma. 9, 559 (2008).

68. $\mathrm{Hu}, \mathrm{Z}$. et al. VisANT 4.0: integrative network platform to connect genes, drugs, diseases and therapies. Nucleic Acids Res. 41, W225-W231 (2013).

\section{Acknowledgements}

We are grateful to Dr. Yadong Huang and Chengzhong Wang for providing the APOE isogenic lines. This work was partially supported by Mayo Clinic Center for Regenerative Medicine, Neuroregeneration Lab. Lab Funding: This work was supported by NIH grants RF1AG051504, R37AG027924, RF1AG046205, RF1AG057181, R01AG066395,

P01NS074969, and P30AG062677 (to G.B.), Younkin Fellowship and Alzheimer's Association Research Fellowship 2018-AARF-592302 (to J.Z.), and R01AG061796 (to N.E.-T.). Dr. Zbigniew K. Wszolek is partially supported by the Mayo Clinic Center for Regenerative Medicine, the gifts from The Sol Goldman Charitable Trust, the Donald G. and Jodi P. Heeringa Family, the Haworth Family Professorship in Neurodegenerative Diseases fund, and by Albertson Parkinson's Research Foundation.

\section{Author contributions}

J.Z., C.L., F.S., N.E.T., T.K., and G.B. conceived and designed the project, and wrote the paper. M.D., L.J., S.G.Y., N.R.G.R., Z.W., and D.B. helped with collecting human skin biopsies and generating iPSC lines. J.Z., Y.F., Y.Y., W.L., Y.M., K.C., L.J., and T.N. executed the experiments and analyzed the data. Y.R., X.W., Y.C., and Y.A. performed analysis for RNA-sequencing data.

\section{Competing interests}

The authors declare no competing interests.

\section{Additional information}

Supplementary information is available for this paper at https://doi.org/10.1038/s41467020-19264-0.

Correspondence and requests for materials should be addressed to G.B.

Peer review information Nature Communications thanks Tracy Young-Pearse and the other anonymous reviewer(s) for their contribution to the peer review of this work. Peer reviewer reports are available.

Reprints and permission information is available at http://www.nature.com/reprints

Publisher's note Springer Nature remains neutral with regard to jurisdictional claims in published maps and institutional affiliations.

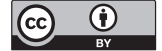

Open Access This article is licensed under a Creative Commons Attribution 4.0 International License, which permits use, sharing, adaptation, distribution and reproduction in any medium or format, as long as you give appropriate credit to the original author(s) and the source, provide a link to the Creative Commons license, and indicate if changes were made. The images or other third party material in this article are included in the article's Creative Commons license, unless indicated otherwise in a credit line to the material. If material is not included in the article's Creative Commons license and your intended use is not permitted by statutory regulation or exceeds the permitted use, you will need to obtain permission directly from the copyright holder. To view a copy of this license, visit http://creativecommons.org/ licenses/by/4.0/.

(c) The Author(s) 2020, corrected publication 2021 